\title{
Road-traffic monitoring by knowledge-driven static and dynamic image analysis
}

\author{
Antonio Fernández-Caballero ${ }^{\text {a,b,*, }}$, Francisco J. Gómez ${ }^{\text {a,b }}$, Juan López-López a \\ ${ }^{a}$ Instituto de Investigación en Informática de Albacete (I3A), Universidad de Castilla-La Mancha, 02071-Albacete, Spain \\ ${ }^{\mathrm{b}}$ Departamento de Sistemas Informáticos, Escuela Politécnica Superior de Albacete, Universidad de Castilla-La Mancha, 02071-Albacete, Spain
}

\begin{abstract}
This article presents a visual application which allows a study and analysis of traffic behavior on major roads (more specifically freeways and highways), using as the main surveillance artefact a video camera mounted on a relatively high place (such as a bridge) with a significant image analysis field. The system described presents something new which is the combination of both traditional traffic monitoring systems, that is, monitoring to get information on different traffic parameters and monitoring to detect accidents automatically. Therefore, we present a system in charge of compiling information on different traffic parameters. It also has a surveillance module for that traffic, which can detect a wide range of the most significant incidents on a freeway or highway.
\end{abstract}

(c) 2007 Elsevier Ltd. All rights reserved.

Keywords: Motion segmentation; Image analysis; Motion estimation; Road-traffic monitoring; Vehicle classification; Vehicle tracking

\section{Introduction}

Since the automobile became a popular phenomenon, the need to watch over the safety of motor vehicle occupants and the rest of the people included in road safety emerged. The proliferation of these moving elements forced governments to take measures to guarantee good driving habits as well as safe driving on the roads where these vehicles traveled. Although the first highway code had already been put into effect, it was obvious that it was not enough to guarantee that the above mentioned requirements (good driving habits and safe driving) would be fully complied with. Other mechanisms had to be put into effect to make traveling as safe as possible at all times.

Until a few years ago, surveillance amounted to no more than the presence of policemen on the roads who informed of any road violations. In the last years, there has been a

\footnotetext{
${ }^{*}$ Corresponding author. Address: Departamento de Sistemas Informáticos, Escuela Politécnica Superior de Albacete, Universidad de Castilla-La Mancha, 02071-Albacete, Spain. Tel.: +34 967599200; fax: +34 967 599224.

E-mail address: caballer@dsi.uclm.es (A. Fernández-Caballero).
}

growing interest in the use of automatic mechanisms capable of providing information about the behavior of automobiles on highways and city roads. The most attractive of these, without a doubt, is video image detection. A survey of video processing techniques for traffic applications (Kastrinaki, Zervakis, \& Kalaitzakis, 2003) demonstrates the great importance of the topic addressed. Video image detection entails the gathering of data which describes the characteristics of the vehicles and their motion. Some examples of such data are the number of vehicles, the speed of the vehicles, vehicle's size, vehicle's length, type of vehicle (car, van, truck or bus). This information is useful for the purposes described next. First, it is a back up tool for law enforcement agencies. It is very useful to be able to detect speed limit violations, reckless driving or illegal use of the bus lane. It is also possible to detect situations of traffic congestion and incidents after locating traffic jams or accidents. Lastly, it would be of great help to be able to increase the capacity of the road-traffic network from the general road information network and guide traffic towards less congested roads.

A typical computer vision system for traffic monitoring consists of a CCD camera, which captures on-line video 
images that are digitalized and stored in a computer. In this computer, some vision algorithms carry out vehicle detection, their tracking and their classification. Notice that every video camera-based traffic control system is classified into two types:

- Traffic monitoring, which includes: (1) Monitoring to obtain information on different traffic parameters, such as: number of vehicles per unit of time, vehicle classification, average speed, individual speed of each vehicle, etc. (2) Monitoring to detect accidents automatically, also called AID (Automatic Incident Detection). These focus on finding irregularities, such as: stopped traffic, slow traffic, traffic jams, etc.

- Traffic control for toll purposes or sanctions. The ones focusing on sanctions control traffic violations and detect vehicles unmistakably. An example is the license plate identifiers. Those focusing on tolls analyze traffic at the toll booths, studying the number of vehicles in each line, average waiting time in line, etc.

This article presents a visual application which allows a study and analysis of traffic behavior on major roads (more specifically freeways and highways), using as the main surveillance artefact a video camera mounted on a relatively high place (such as a bridge) with a significant image analysis field. It is a traffic monitoring system which allows the gathering of some traffic parameters and the detection of some of the most significant and frequent incidents that can take place on a freeway or highway. Our approach is similar to (Cucchiara, Piccardi, \& Mello, 2000) in the sense that image-processing modules extract visual data from the scene by spatio-temporal analysis and high-level modules are designed to work on vehicles and their attributes and to exploit expert knowledge on traffic conditions.

\section{Road-traffic monitoring approaches}

A great number of road-traffic monitoring systems are based on motion detection to segment the regions of the image. As an example, consider VISATRAM ( $\mathrm{Zhu}, \mathrm{Xu}$, Yang, Shi, \& Lin, 2000), a system for automatic traffic monitoring using 2D spatio-temporal images. A TV camera is mounted above a highway to monitor the traffic through two slice windows, and a panoramic view image and an epipolar plane image are formed for each lane. If the regions contain the appropriate characteristics, vehicles are considered and can be counted or tracked as desired. Out of the motion detection techniques defined so far, the two most frequently used in road-traffic monitoring are the image difference method and the motion detection technique based on features.

Image difference (Sonka, Hlavac, \& Boyle, 1993) is based on the fact that the differences between two frames captured at different time instants reveal regions in motion. An image difference $I$ is generated by calculating the abso- lute difference between two frames $\left(I_{1}\right.$ and $\left.I_{2}\right)$ and thresholding the result.

$$
I(x, y)= \begin{cases}0, & \text { if }\left|I_{1}(x, y)-I_{2}(x, y)\right| \leqslant \theta \\ 1, & \text { otherwise }\end{cases}
$$

where $\theta$ is an appropriate threshold. In the case of traffic monitoring, it is usual for $I_{1}$ to be the input frame and $I_{2}$ to be the reference frame (background). The reference frame is an image from the scene, without vehicles. The purpose of the threshold is to reduce the effects of noise and changes in the scene's lighting. The latter is a great problem in computer vision and it is usually necessary to use methods of dynamic updating of the reference frame to adapt to the scene's lighting. In the recent works of our research team (e.g. López, Fernández-Caballero, Fernández, Mira, \& Delgado, in press; López, Fernández-Caballero, Fernández, Mira, \& Delgado, 2006; López, Fernández-Caballero, Mira, Delgado, \& Fernández, 2006) the image difference is performed on two consecutive image frames. Some other works (Dubuisson \& Jain, 1995; Gil \& Punt, 1993; Teal \& Ellis, 1996; Waterfall \& Dickinson, 1984) also describe systems with motion analysis based on simple frame differencing. To solve the problem of using an accurate background image, a self-adaptive background approximating and updating algorithm based on optical flow theory has recently been presented for traffic surveillance (Ji, Wei, \& Feng, 2006). To detect the moving regions of interest in the scene, the difference image between the current frame and the updating background is first obtained by using a color image difference model, and then a self-adaptive thresholding segmentation method for moving object detection based on the Gaussian model is developed and implemented. Motion detection based on characteristics (Sonka et al., 1993) looks for the most important features frame by frame. The first step consists of identifying the appropriate feature. Once identified in the frames, a matching procedure must be done to find the correspondence from among those points in the following frames. For instance, the feature information is extracted by the seed-fillingbased method and is presented to the input of neural network for vehicle detection and classification (Ha, Lee, \& Kim, 2004).

Some complete traffic monitoring systems are available to date. The TRIP (Traffic Research using Image Processing) system (Dickinson \& Wan, 1989; Inigo, 1985) is designed to count vehicles traveling in a two-lane, twoway highway. The camera is placed in the highway, looking vertically onto the scene. By using a reference image, the input images are differentiated and the result thresholded. This leads to a binary image where vehicles in motion should appear. Another system (Takaba, Sakauchi, Kaneko, Hwang, \& Sekine, 1982) uses sampling points capable of detecting the presence of a vehicle with the purpose of counting the number of vehicles. By placing two sampling points and calculating the time spent in the detec- 
tion of the vehicle between the two points, the speed of the vehicles can be measured. Detection lines (Abramczuk, 1984) can be used instead of sampling points. These allow the vehicles to be counted and to obtain the approximate speed of the vehicles. The Wide Area Detection System (WADS) system is capable of detecting, counting and measuring the speed of the vehicle in motion (Inigo, 1989). This system works in a reduced area of the image, that is, a horizontal line on a maximum of three lanes for vehicle detection. Speed is obtained by tracing about thirty lines of the highway, at regular intervals. Moreover, the TITAN system, which worked in real time in a microcomputer and was completed in April 1990, was developed by the Traffic Control and Analysis Department of the French company INRETS (Blosseville, Krafft, Lenoir, Motyka, \& Beucher, 1989; Blosseville, 1990). This system used morphological markers. The different parts of a car (rood, hood, etc.) were identified through morphological filtering. The topological relationship between them enabled vehicle detection.

Motion detection by frame differentiation is also the nucleus of a system capable of counting vehicles, measuring their speed and tracking them in complex highway crossings (Ali \& Dagless, 1991, 1992; Bulas-Cruz, Ali, \& Dagless, 1993). The commercial system CCATS (Camera and Computer Aided Traffic Sensor) uses a CCD camera placed in a highway, looking vertically with a $25 \mathrm{~m}$ visibility (Briquet, 1992; Versavel, Lemaire, \& van der Stede, 1989). The whole image is digitized, but only about twenty pixels per rail are processed. This system is capable of working by day, as well as by night. IMPACTS system (Hoose, 1989; Kelly, 1992) operates at a macroscopic level, offering a qualitative description of the space distribution of moving and stationary traffic in the scene. The system divides the image into cells, with each cell taking the approximate width of a lane and longitude of a car. Each cell may be in one of the following states: no vehicle, stationary vehicle or moving vehicle. Another system, capable of measuring traffic queue parameters (Fathy \& Siyal, 1995), operates in small regions of the image. The presence of vehicles together with lack of motion means a queue. This condition is tested at all intervals to calculate the queue length. Another system, capable of detecting and tracking multiple vehicles and, at the same time, giving information to classify them uses Kalman filters (Koller, Weber, \& Malik, 1994; Koller, Weber, Huang, et al., 1994; Malik, Weber, Luong, \& Koller, 1995). The same system has been developed, incorporating a common motion restriction (Beymer, McLauchlan, Coifman, \& Malik, 1997). A method has also been developed using the image difference technique to segment moving vehicles. This method automatically generates a reference image (Cornish \& Wakefield, 1996), creating a region of interest which reduces the parts of the image where vehicles can be found, thus reducing computation costs. Another system (Barni, Bartolini, Capellini, Lambardi, \& Piva, 1998) uses the differences between consecutive image pairs to ini- tialize a level field which is processed by a probabilistic motor based on heuristic rules. This system classifies each pixel into one of these four categories: moving, still, uncovered and covered, according to the position that the vehicles occupy on the road in the current image. Through this, the limits for each object are established and an estimate of the speed of the vehicles is obtained. A system has been developed at Jaime I University in Castellón (Badenas, Sanchiz, \& Pla, 1999; Badenas, Bober, \& Pla, 2001) which is capable of detecting a vehicle's path, as well as reporting traffic conditions by applying segmentation and Kalman filters.

The greatest effort in the development of ITS was probably made by the U.S. Department of Transportation when it requested Mitretek Systems, Inc. (Proper, 1999) to carry out several ITS projects. The ones that stand out are those meant for a metropolitan ITS infrastructure, to which the following systems belong: Arterial Roads Control System, Highway Control System, Transit Control System (meant for public transportation in cities), Incident Control System, Emergency Control System, Electronic Collection System for Toll Roads (freeways), Electronic Fares Payment System (in cities), Intersections between Freeways and Railroad Tracks, Regional Information for travelers and Integrated Systems. The subject continues to arouse a lot of interest, as can be seen in the article on vehicle detection and classification (Gupte, Masoud, Martin, \& Papanikiolopoulos, 2002), where a six-stage system is proposed: (1) segmentation, (2) region tracking, (3) recovery of vehicle parameters, (4) vehicle identification, (5) vehicle tracking and (6) vehicle classification.

\section{Static and dynamic image analysis for road-traffic monitoring}

\subsection{Road-traffic monitoring architecture}

As stated before, the objective of this article is to present a visual system which controls traffic in freeways and highways. Besides, the system has a new feature which is the combination of both traditional traffic monitoring systems into one. That is to say that we show a system which is in charge of taking information on a number of traffic parameters. It also has a surveillance module for that traffic which is capable of detecting a wide range of the most significant incidents in a freeway or highway.

In this case, the first stumbling task, in order to put this system into practice, is the camera's position. This is so because the traffic parameter monitoring systems require the camera to be very slanted, taking the images practically at right angles to the road, whereas the AID systems require a field of vision of one-hundred to five-hundred meters wide for reasonable vehicle tracking. We have decided to compromise on a field of vision of $100-150 \mathrm{~m}$ long. As an initial limitation, we have the fact that the application is capable of controlling three lanes of normal 
traffic flow and one entrance or exit ramp, at the most. All these lanes go in the direction of traffic, which is from where the camera is towards the horizon.

The system is capable of identifying each vehicle that appears in each image and it assigns them the type of vehicle they belong to and the real situation they occupy on the road. The types of vehicles the application can detect are: motorcycles, cars, passenger and cargo vehicles (van) and heavy vehicles (there is no distinction between trucks and buses). At the same time, it recognizes objects which do not belong to these stereotypes. These objects are assigned to class "Foreign object". Once a vehicle is detected (Fernández, Fernández-Caballero, López, \& Mira, 2003; Mira, Delgado, Fernández-Caballero, \& Fernández, 2004), it is followed closely through the images captured by the video camera, so that each vehicle is tracked down from the time it enters the scene until it leaves it. Through this tracking, we get information from that vehicle regarding traffic parameters, such as speed, number of vehicles of each type that have entered the scene, location and speed of each vehicle, as well as the detection of any incidents on the road. Incident detection is carried out by two modules integrated into the system. These modules are called "Static Surveillance" and "Dynamic Surveillance", respectively, depending on how they analyze the current image or the comparison of that image with the previous one. Another feature of the application is letting the user determine which incidents he/she wants to control at all times. The group of incidents that can be controlled by the application is shown in Table 1.

At the end of the image sequence processing, the system is in the position to offer the following detailed list of parameters:

- Total number of motorcycles, cars, vans and heavy vehicles (trucks and buses) traveling on the road during the processing period.
- Total number of vehicles traveling in the right and left hand lanes and middle lane, if there is one.

- Total number of vehicles traveling in the entrance/exit ramp, if there is one.

- Total number of vehicles traveling on the road.

- Average speed of traveling motorcycles, cars, vans and heavy vehicles.

- Average speed of vehicles traveling in the right and left hand lanes and middle lane, if there is one.

- Average speed of vehicles traveling in the entrance/exit ramp, if there is one.

- Average speed of all vehicles traveling on the road.

The system uses a camera mounted on a bridge which captures real traffic images. These images, which are processed in a 256-color-gray scale format, are segmented according to (Fernández-Caballero, Mira, Fernández, \& López, 2001; Fernández-Caballero, Mira, Delgado, \& Fernández, 2003; Fernández-Caballero, Fernández, Mira, \& Delgado, 2003) model in such a way that each object's movement captured in the real image may be observed. The segmented image shows the image's background in black, whereas the motion detected in the vehicles is represented by pixels in different black gray scales (see Fig. 1b). A great number of pixels can be seen in gray scales which do not represent moving objects; those pixels constitute the image's noise. This application analyzes the sequence of segmented images, giving rise to a sequence of processed images, where each vehicle is shown through an identifying color which is maintained while the vehicle travels along the area of analysis. The shape of the vehicle is rectangular (see Fig. 1c).

The extraction of the moving objects in the segmented image has been called static analysis, since it is based on the study of a single segmented image. Afterwards, a dynamic analysis is done based on the comparison between the information from the vehicles in the current segmented

Table 1

Incidents

\begin{tabular}{|c|c|c|}
\hline Name of incident & What is being controlled? & Who controls it? \\
\hline Foreign object & The presence of foreign objects on the road that do not belong to any valid type of vehicle & $\begin{array}{l}\text { Static } \\
\text { surveillance }\end{array}$ \\
\hline Vehicle on shoulder & $\begin{array}{l}\text { A vehicle traveling on the shoulder or the median. For all practical purposes, the median is considered the } \\
\text { shoulder }\end{array}$ & $\begin{array}{l}\text { Static } \\
\text { surveillance }\end{array}$ \\
\hline $\begin{array}{l}\text { Heavy vehicle on } \\
\text { restricted dates }\end{array}$ & Heavy vehicles traveling on restricted dates & $\begin{array}{l}\text { Static } \\
\text { surveillance }\end{array}$ \\
\hline $\begin{array}{l}\text { Driving too fast or too } \\
\text { slowly }\end{array}$ & $\begin{array}{l}\text { The speed of each vehicle is calculated. It is then determined whether it is within the maximum and } \\
\text { minimum speed limits. The speed limits established for each type of vehicle, as well as the maximum and } \\
\text { minimum limits established for each lane will be controlled. }\end{array}$ & $\begin{array}{l}\text { Dynamic } \\
\text { surveillance }\end{array}$ \\
\hline $\begin{array}{l}\text { Stopped vehicle on the } \\
\text { road }\end{array}$ & A vehicle stopped in any part of the road that is being analyzed & $\begin{array}{l}\text { Dynamic } \\
\text { surveillance }\end{array}$ \\
\hline Traffic congestion & An excessive number of vehicles stopped on the road. This limit can be set by the user & $\begin{array}{l}\text { Dynamic } \\
\text { surveillance }\end{array}$ \\
\hline Overstepping a solid line & Vehicles overstepping a solid line which delimits two traffic lanes & $\begin{array}{l}\text { Dynamic } \\
\text { surveillance }\end{array}$ \\
\hline
\end{tabular}



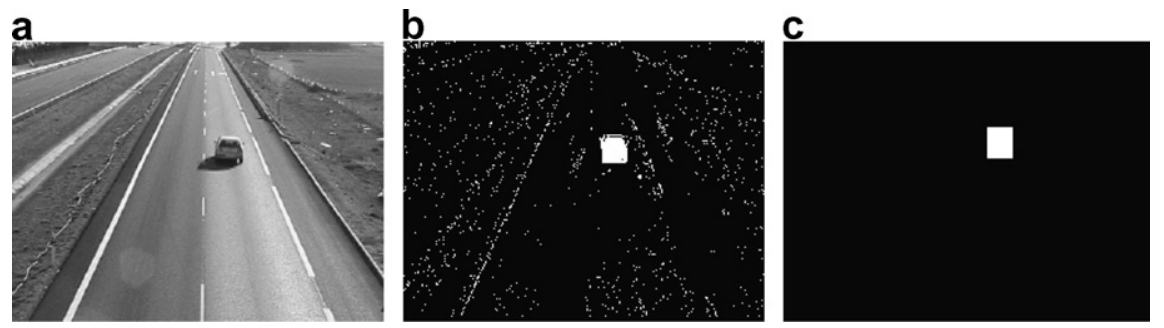

Fig. 1. Road-traffic monitoring images. (a) Real image in gray scales. (b) Segmented image. (c) Processed image.

image and the previous one. This whole process is carried out periodically with a $0.5 \mathrm{~s}$ frequency.

Notice that the application will take three inputs and has three data outputs (see Fig. 2). The inputs are:

(1) Segmented Image at cycle $i$ - refers to the segmented image corresponding to the execution cycle where the application is located.

(2) Reference Image - as aforementioned, provides information about the characteristics of the road.

(3) Vehicle data at cycle $i-1$ - refers to the information about the vehicles in the segmented image analyzed in the previous execution cycle (cycle $i-1$ ). This information refers to the type of vehicle, dimensions and situation of each vehicle that appeared in the previous image. Naturally, this information would not be available in the first execution cycle.

The application's outputs are:

(1) Processed Image at cycle $i$ - the image generated through the tracking of each vehicle in the area of analysis. This information will be used by the following execution cycle.

(2) Vehicle data at cycle $i$ - refers to the information taken from the analysis of the current segmented image about the vehicles that appear in it. This information will be relevant in the following execution cycle. This information refers to the type of vehicle, dimension and situation of each vehicle in the area of analysis.

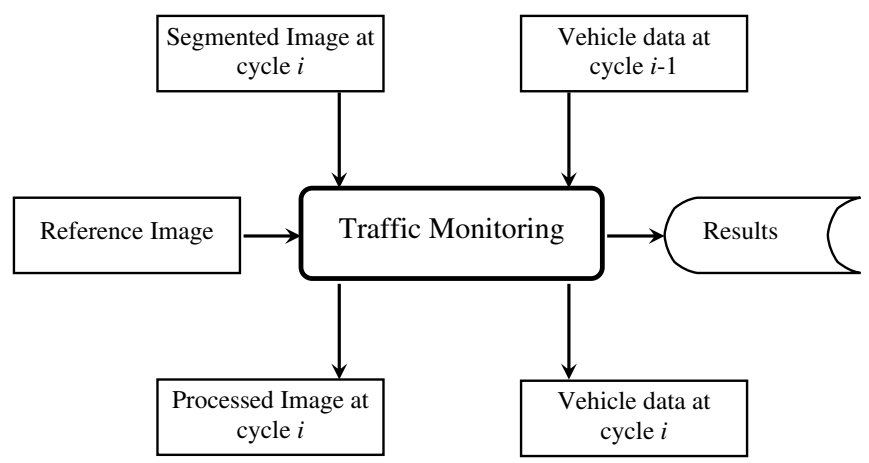

Fig. 2. "Traffic Monitoring" module.
(3) The results obtained from the Static and Dynamic Analysis - controlled traffic parameters as well as incidents detected. This information is available for subsequent reference.

Fig. 3 represents a more detailed level of the structure of module "traffic monitoring". Notice that module "Traffic Monitoring" is broken down into three big tasks: static analysis, dynamic analysis and results generator. These tasks are explained in detail in Table 2. On the other hand, the new information in the tasks of this module is defined in Table 3.

\subsection{Static analysis for road-traffic monitoring}

Next, we will list the performance and the different parts of the static analysis. First, we will show a diagram of this task in Fig. 4. Notice that static analysis is broken down into the "Image Processing" and "Static surveillance" tasks.

\subsubsection{Image processing}

This task uses "Segmented Image at cycle $i$ " and "Reference Image" as inputs and "Static Traffic Parameters" as output. The "Image Processing" task is broken down into two processes: Moving Object Detection and Vehicle Classification. To detect moving objects in the scene, the

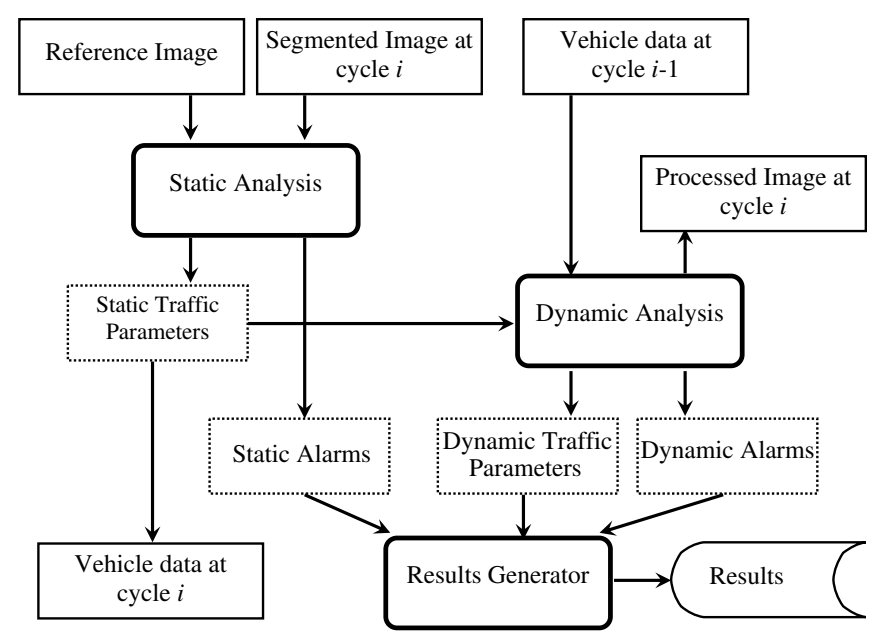

Fig. 3. Detailed structure of the "traffic monitoring" module. 
Table 2

Functionalities of the "traffic monitoring" tasks

\begin{tabular}{ll}
\hline Task & Module's function \\
\hline Static & $\begin{array}{l}\text { It is in charge of the sequence path of the current } \\
\text { segmented image, eliminating noise from it and extracting } \\
\text { information from moving objects. It later examines which } \\
\text { of these objects belong to valid types of vehicles. It } \\
\text { calculates the dimensions and position of each vehicle. It } \\
\text { controls the incidents which can be determined by } \\
\text { studying the current image }\end{array}$ \\
Dynamic & $\begin{array}{l}\text { It is in charge of comparing the information gathered } \\
\text { from the vehicles in the segmented image from the } \\
\text { previous cycle with the information gathered from the }\end{array}$ \\
& $\begin{array}{l}\text { static analysis of the current cycle. It is in charge of } \\
\text { matching previous and current vehicles. It calculates each } \\
\text { vehicle's speed. It determines whether a vehicle has gone } \\
\text { in or out of the area of analysis. It controls incidents that } \\
\text { occur as a consequence of the vehicles' motion }\end{array}$ \\
Results & $\begin{array}{l}\text { It gathers all the information provided by the other two } \\
\text { tasks and gives them the proper format to be stored in the }\end{array}$ \\
& Results data base
\end{tabular}

algorithms described in Fernández-Caballero, Fernández, et al. (2003) are performed. Once a moving object have been detected, the dimensions of the object are established, its center is calculated and the coordinates of its center are extrapolated to the "Reference Image", which returns the value of the colors of those coordinates. Those colors are related to the position the object occupies, according to Table 4, and are associated to the "Reference Image" (see example in Fig. 5b). Notice that the coordinates of the center of the object are the lower boundary and the half point between the left and right boundaries. This is done this way so that the center can have better correspondence with the real situation of the vehicle.

Thus, through the task Moving Object Detection the number of moving objects in the area of analysis at the time is determined, as well as their dimensions, and position on the road. This is information Moving Objects. The process Vehicle Classification determines the objects whose established position is not "Out of bounds". A vehicle which inherits the characteristics of the object is created and the object is eliminated. The vehicle is classified, according to its type. The type of vehicles established as valid vehicles are:

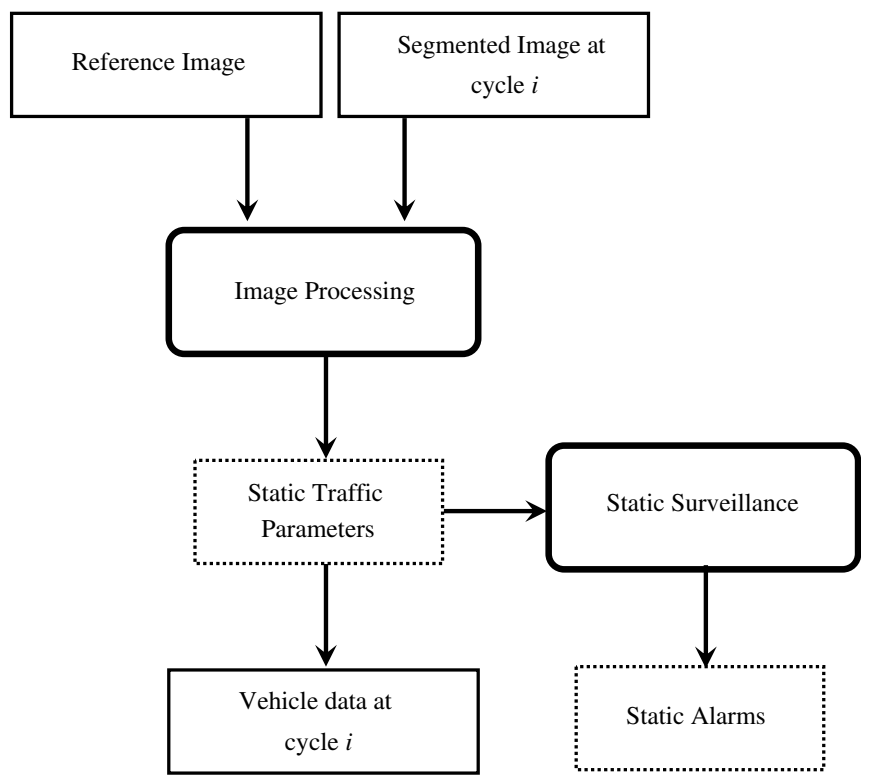

Fig. 4. Static analysis structure.

- Motorcycle,

- Car,

- Van,

- Heavy Vehicle (both trucks and buses are included).

If the object does not belong to any type, it is labeled as a "Foreign object". Once the vehicle is classified, an estimate of its minimum and maximum position is calculated in the following image, depending on its minimum or maximum speed, respectively. All this information is called "Static Traffic Parameters" and it goes on to the Dynamic Analysis as such and to the following execution cycle under the name of "Vehicle data at cycle $i$ ".

To know a vehicle's dimensions and the distance between vehicles, three "Distance Zones" have been defined which indicate three areas where the value of the resolution of each pixel, length and crosswise, is going to be the same for that whole area but different from the other areas. The user indicates this area so that the pixels in each distance zone represent a number of centimeters which is lower than the pixels from an area further away from the

Table 3

New information at "traffic monitoring" module

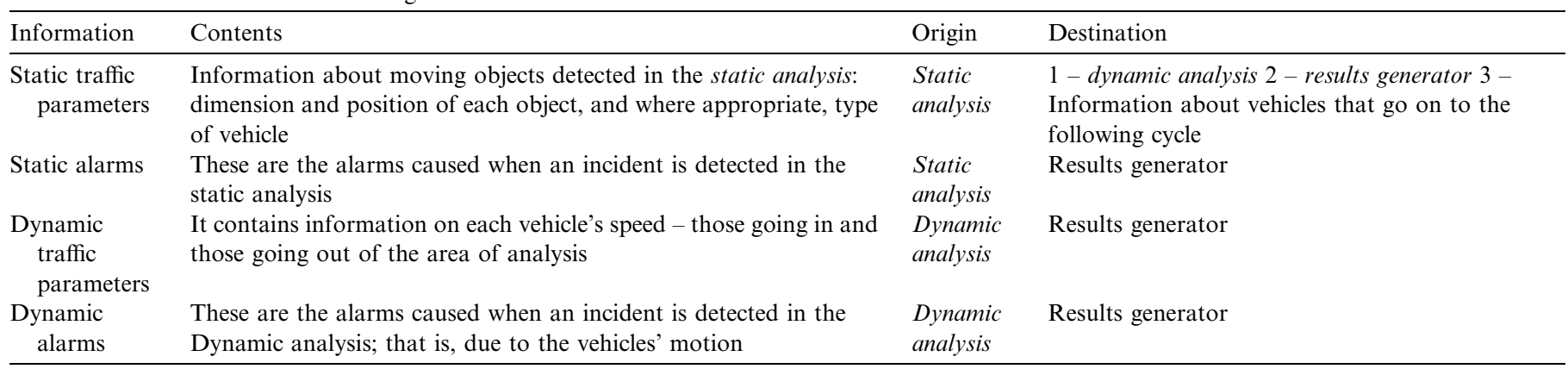


Table 4

Colors table

\begin{tabular}{lll}
\hline Color & $\begin{array}{c}\text { Color } \\
\text { (byte) }\end{array}$ & Area that it represents \\
\hline White & 255 & Reserved \\
Yellow & 252 & Entrance/exit ramp \\
Red & 224 & Shoulder \\
Dark Gray & 146 & Right lane \\
Light Blue & 31 & Left lane \\
Green & 28 & Middle lane \\
Dark Blue & 3 & "Out of bounds" (it does not belong \\
& & to the road) \\
Black & 0 & Lines and dividing lines \\
\hline
\end{tabular}

camera. An example of distance zones for the "Reference Image" is found in Fig. 5c:

\subsubsection{Static surveillance}

This task is capable of controlling three incidents through the study of the object's/vehicle's dimension and its position, as shown in Table 5.

The information called "Static alarms" is also generated. For each vehicle detected:

- the position that the vehicle occupies is checked. If it is on the shoulder, a record is kept informing that the vehicle is traveling on the shoulder,

- the type of vehicle is checked, and:

- if it is a heavy vehicle, the date is checked to see if it is traveling on restricted dates;

- if it is a "Foreign object", a record is kept indicating that an unidentified object remains in the area of analysis.

\subsection{Dynamic analysis for road-traffic monitoring}

The study of an isolated image, obviously, does not contain all the information needed. That is why the study of two consecutive images is necessary in order to see what has happened in between images. This process is called dynamic analysis (see Fig. 6). The dynamic analysis is bro-
Table 5

Static incidents

\begin{tabular}{ll}
\hline $\begin{array}{l}\text { Static } \\
\text { Incident }\end{array}$ & How is it controlled? \\
\hline $\begin{array}{l}\text { Vehicle } \\
\text { traveling } \\
\text { along } \\
\text { shoulder }\end{array}$ & $\begin{array}{l}\text { The coordinates in the center of the vehicle are } \\
\text { extrapolated to the reference image. Depending on the } \\
\text { color of the pixel corresponding to those coordinates, the } \\
\text { vehicle will be in one area or another. If the pixel is red, } \\
\text { the vehicle is traveling on the shoulder or median }\end{array}$ \\
$\begin{array}{l}\text { Destricted } \\
\text { traffic }\end{array}$ & $\begin{array}{l}\text { During those dates when traffic is restricted, the traveling } \\
\text { of vehicles in the "heavy vehicles" category is controlled. }\end{array}$ \\
& $\begin{array}{l}\text { It is necessary for the user to have previously defined the } \\
\text { restriction dates. The task "Static surveillance" is in } \\
\text { charge of comparing those dates with the current date }\end{array}$ \\
& $\begin{array}{l}\text { and whether any of the objects detected has "heavy } \\
\text { vehicle" characteristics or not }\end{array}$ \\
The existence of objects detected that do not belong to \\
Foreign \\
objects
\end{tabular}$\quad$\begin{tabular}{l} 
any valid type of vehicle is checked \\
\hline
\end{tabular}

ken down into four tasks: "Image Comparison", "Vehicle Tracking", "Traffic Parameter Calculation" and "Dynamic Surveillance".

\subsubsection{Image comparison}

This is the fundamental part of the dynamic analysis. It is in charge of finding the differences between an image and the previous one. This is done by comparing the information obtained from the Static Analysis of the vehicles in the current segmented image, located in "Static Traffic Parameters", and information "Vehicle data at cycle $i-1$ ", which contains static information from vehicles in the segmented image included in the previous cycle. The task is broken down into two processes: Vehicle relation and Vehicle classification. The new information is itemized in Table 6.

In task "Image Comparison", the first thing that is calculated is the combinatory relationship of each vehicle in the previous image with each vehicle in the current image. This process is based on weight classification for each relationship, according to the following criteria. It is essential for the current vehicle to be further ahead than the other one, in the direction of traffic. If this is so, then:

- The weight established in parameter Advanced Position is established in the total weight of this relationship. This weight is expressed as $W_{\mathrm{AP}}$.
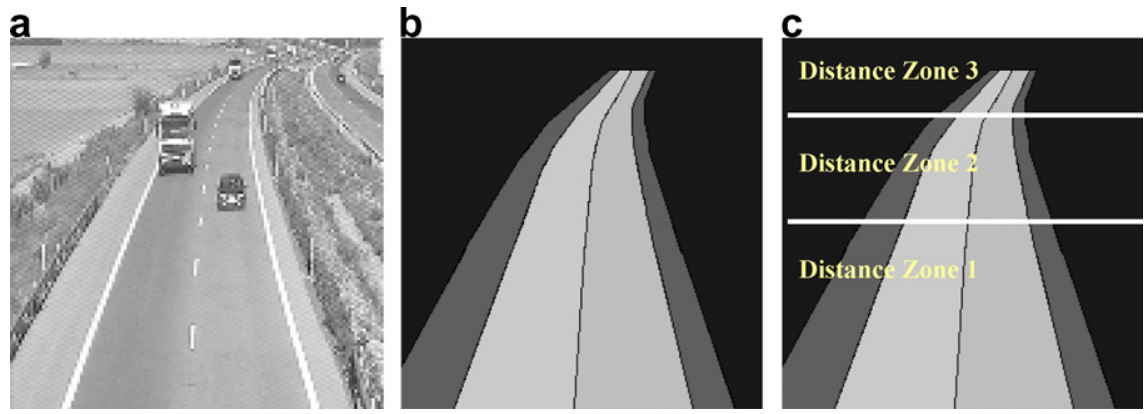

Fig. 5. Example of reference image. (a) Road-traffic image. (b) Reference Image. (c) Distance zones. 


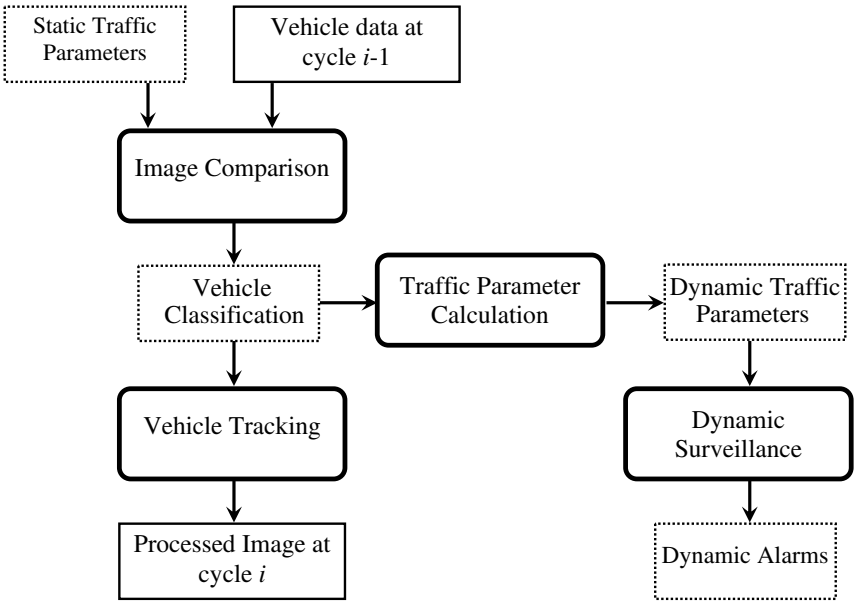

Fig. 6. Dynamic analysis structure.

- If they are in the same lane, the weight established in characteristic Same Lane is added to the total weight of the relationship. The weight for this argument is called $W_{\mathrm{SL}}$.

- If both vehicles belong to the same type, the weight established in Same Type is added to the total weight of the relationship. This weight is called $W_{\mathrm{ST}}$.

- If the current vehicle is within the position estimates done for the previous vehicle, and it was within the speed limits, the weight established in parameter Within Speed Limits is added to the total weight of the relationship. This weight is called $W_{\mathrm{WSL}}$.

If the characteristics of any parameter are not fulfilled, that weight is, naturally, not added to the total weight of the relationship. Therefore, the total weight of a combinatory relationship is called $\mathrm{TW}_{\mathrm{CR}}$ and it is defined as

$\mathrm{TW}_{\mathrm{CR}}=W_{\mathrm{AP}}+W_{\mathrm{SL}}+W_{\mathrm{ST}}+W_{\mathrm{WSL}}$

Once all possible combinatory relationships have been established and the weight of those relationships has been determined, we go on to the final classification between vehicles (Vehicle classification) from the previous and current images. In this process, the combinatory relationships established are compared and a vehicle from the previous image is matched with one from the current image through the selection of the relationship with the greatest possible weight. This is done in such a way that if a match has been established between a previous and a current vehicle and a subsequent relationship containing the same current vehicle with greater weight is found:

(1) the previous match is undone,

(2) a new match is established for the current vehicle with the previous vehicle from the relationship with the greatest weight,

(3) another match is tried for the previous vehicle through the search of another relationship which will contain it.

Once this process is completed, the final classifications between vehicles from the previous and current images are obtained. This means that the previous and current vehicles which are matched represent the same real vehicle. There might be previous or current vehicles unable to be matched. This condition can correspond to five different situations, as listed in Table 7.

Table 6

"Image Comparison" intermediate information

\begin{tabular}{llll}
\hline Information & What does it contain? & Origin & Destination \\
\hline $\begin{array}{l}\text { Combinatory } \\
\text { relationships }\end{array}$ & $\begin{array}{l}\text { Information on the connection between a previous vehicle and } \\
\text { the current ones } \\
\text { and the total weight of each relationship }\end{array}$ & Vehicle relation & Vehicle classification \\
Vehicle classification & $\begin{array}{l}\text { Information on the final classification. A final classification is } \\
\text { the relationship } \\
\text { with the greatest possible weight between a previous and a } \\
\text { current vehicle }\end{array}$ & $\begin{array}{l}\text { Vehicle } \\
\text { classification }\end{array}$ & $\begin{array}{l}\text { 1- Vehicle tracking, 2- Traffic parameter } \\
\text { calculation }\end{array}$ \\
\end{tabular}

Table 7

Situations of an unmatched vehicle

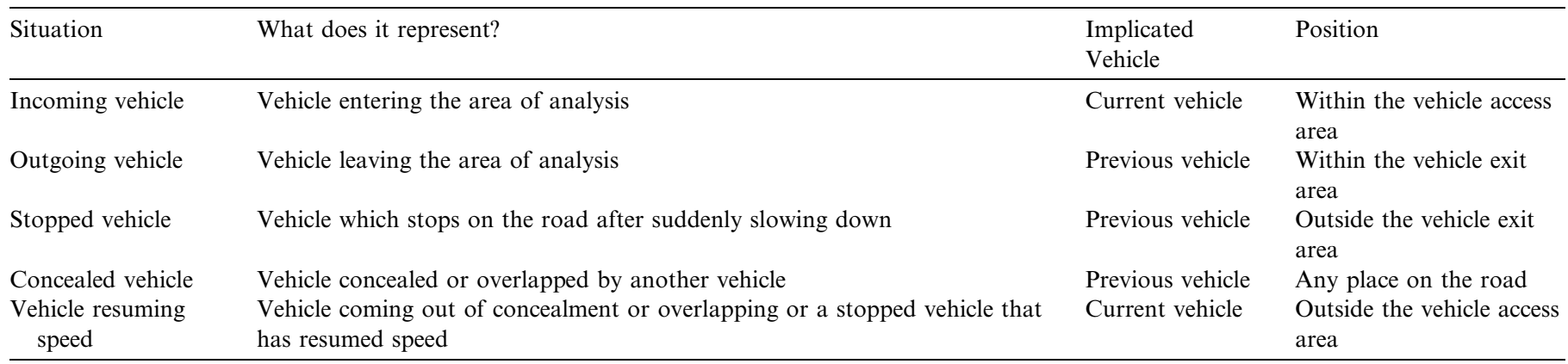




\subsubsection{Vehicle tracking}

When the final matching is done and we know which vehicle from the previous image corresponds to which from the current image, the following operations are carried out:

(1) A color tag is assigned to the current vehicle, depending on:

(a) if the current vehicle is matched to a previous vehicle, the tag from the previous vehicle is assigned to the current one.

(b) if the current vehicle is not matched to a previous vehicle, it is assigned a new color tag.

(2) The vehicle is drawn in the processed image.

\subsubsection{Traffic parameter calculation}

In this task, the speed of the vehicles is calculated and the traffic parameter counters are updated, according to the vehicles that have entered the scene and considering Table 7. For every final match, information about the coordinates of the center of the previous and current vehicles of the match is extracted. The distance traveled by the vehicle is calculated, according to the compromise area where it is situated and where it was situated before, taking into account the resolution of the pixels in the distance zones. Since the distance traveled between two images and the actual period of time between those two images are known, the speed at which the vehicle has been traveling during that time is calculated. The result of the operation in this task is the "Dynamic Traffic Parameters", defined in Table 3.

\subsubsection{Dynamic surveillance}

Using the previous information, the "Dynamic Surveillance" task can start doing his/her job of detecting irregularities linked to object motion. When an incident is detected, the controller is informed. Moreover, information "Dynamic alarms" is created, which contains all the incidents detected in the task "Dynamic Surveillance" in this execution cycle. That information contains the following:
- Date and time of the incident.

- Type of incident.

- Type of vehicle involved, as well as the assigned tag.

- Place on the road where the incident has taken place.

The incidents detected by this task, as well as the way to control them, are enumerated in Table 8.

First, the value of the speed of each current vehicle is extracted. If that speed is less than the minimum corresponding speed, an indication that there has been an incident involving lack of speed is stored in a data structure. If the speed is greater than the maximum corresponding limit, information about an incident involving excessive speed is stored. Later, all the final matches are checked over, and:

- The coordinates from the center of the previous and current vehicle implicated in the match are recorded. The longitudinal distance and the transverse length traveled by the vehicle are calculated. If the vehicle's movement is mainly transverse in the scene, an incident involving a strange movement is indicated.

- The segment from the intermediate line which separates the coordinates from both centers is checked over. If three consecutive black pixels are found, an incident of solid line overstepping is reported.

All previous unmatched vehicles are gone through. If any of them is stopped, an incident involving a stopped vehicle is reported. This means that the counter for the number of stopped vehicles increases by one and if that counter is equal to or greater than the threshold established, information about an incident involving a traffic jam is stored.

\subsection{Results generator}

Its duty is to gather the information provided by the modules static analysis ("Static traffic parameters" and "Static alarms") and dynamic analysis ("Dynamic traffic parameters" and "Dynamic alarms") and give them the

Table 8

Dynamic incidents

\begin{tabular}{|c|c|}
\hline Dynamic incident & How is it controlled? \\
\hline $\begin{array}{l}\text { Traveling too slowly or too } \\
\text { fast }\end{array}$ & $\begin{array}{l}\text { If the speed of the current vehicle exceeds the minimum between the highest speed limit for that type of vehicle and the } \\
\text { highest limit for the lane where the vehicle is currently traveling in, an incident involving excessive speed is detected, that is, } \\
\text { if the speed is below the maximum between the lowest speed limit for that type of vehicle and the lowest limit for the lane } \\
\text { the vehicle is currently traveling in, an incident involving a vehicle traveling too slowly is detected }\end{array}$ \\
\hline Overstepping a solid line & $\begin{array}{l}\text { The reference image is checked to see that there is no solid line established between the position of the previous and current } \\
\text { vehicles. To do this, we go through the middle row between the centers of both vehicles and from the column of one of them } \\
\text { to that of the other. We also check to see if there are three consecutive black pixels }\end{array}$ \\
\hline Traffic congestion & When the number of stopped vehicles is greater that the threshold set up, it is considered a traffic jam \\
\hline Strange movement & $\begin{array}{l}\text { An object moves in such a way that its transverse movement is greater than its longitudinal one. The longitudinal } \\
\text { movement is the number of rows of pixels which separates the previous from the current vehicle. The transverse movement } \\
\text { is the number of columns which separates the two }\end{array}$ \\
\hline
\end{tabular}




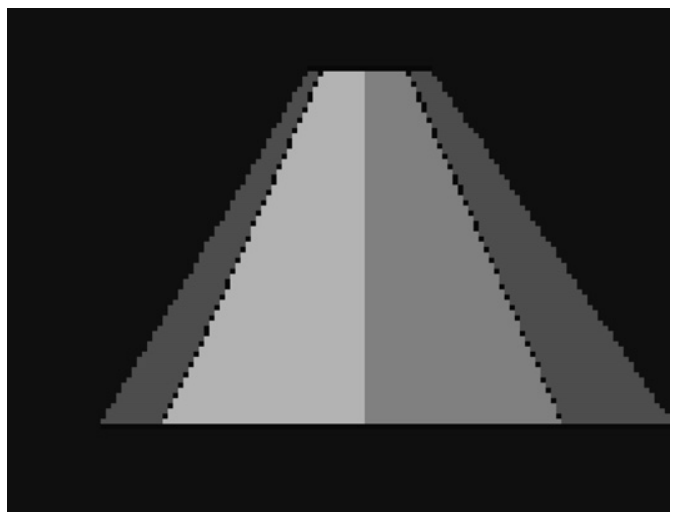

Fig. 7. Reference image for the first three study cases. proper format to be stored in a data base. This way, there is physical record of the whole application control process.

\section{Data and results}

\subsection{First study case: speed calculation I}

In this example, we study the running of the application by taking a controlled vehicle traveling at a constant speed of $100 \mathrm{~km} / \mathrm{h}$ through the area of analysis. The weather conditions that day were pretty unfavorable since there were clouds at all times, which affected the brightness in the scene. We must also point out the presence of strong winds, which caused unwanted movements in the video camera. The reference image for the scene is shown in Fig. 7.

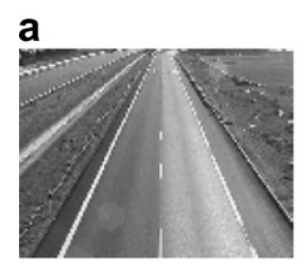

b
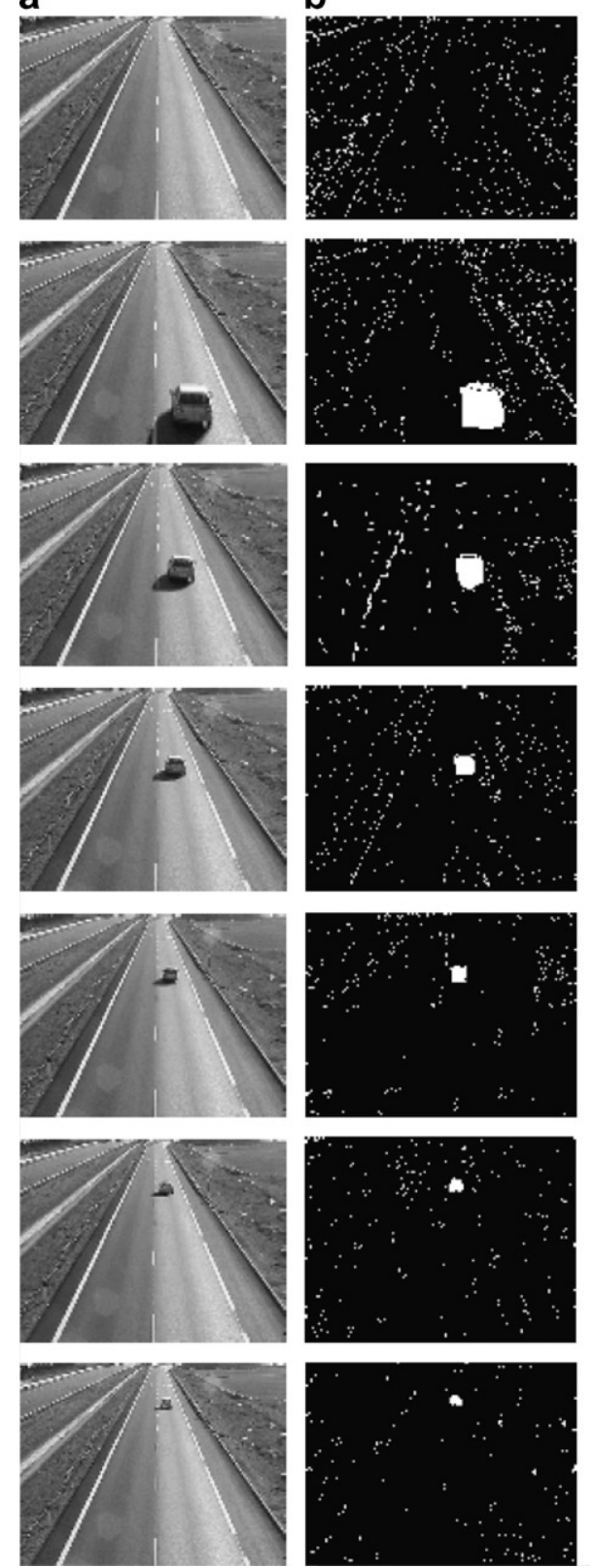

C

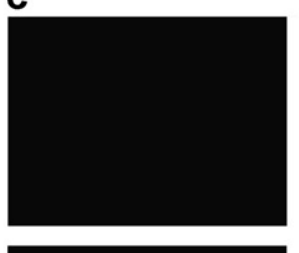

Images at $\mathrm{t}=0 \mathrm{~s}$

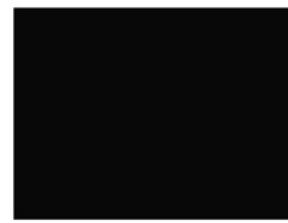

Images at $\mathrm{t}=0,5 \mathrm{~s}$

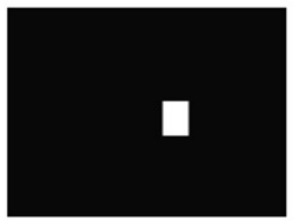

Images at $\mathrm{t}=1 \mathrm{~s}$

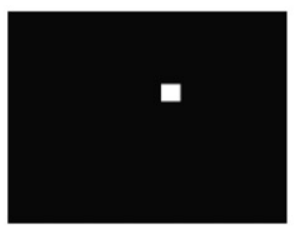

Images at $\mathrm{t}=1,5 \mathrm{~s}$

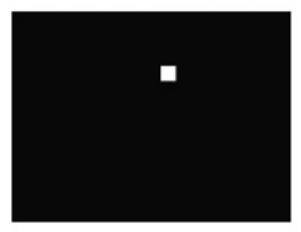

Images at $\mathrm{t}=2 \mathrm{~s}$

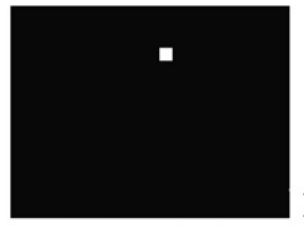

Images at $\mathrm{t}=2,5 \mathrm{~s}$

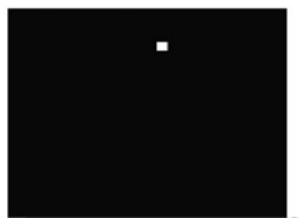

Images at $\mathrm{t}=3 \mathrm{~s}$

Fig. 8. Example 1: speed calculation I. 
Notice that the area of analysis is the area with a color other than dark blue. Only those vehicles whose center is in this area will be considered. The area of analysis has an approximate length of $100 \mathrm{~m}$. We now go on to show the real image sequences in segmented and processed gray scales in order to get a complete vision of the process. This is shown from left to right: segmented and processed real image for each moment of time (see Fig. 8).

Also notice that the frequency between images is half a second, as stated earlier. On the other hand, there is no vehicle to be processed until the third image because, although there is an object in the second segmented image, it is not in the area of analysis established by the reference image. A vehicle belonging to the car type, traveling in the right lane at $100 \mathrm{~km} / \mathrm{h}$, has been detected. The type of vehi- cle and the lane where it was traveling has been detected correctly. Likewise, the speed calculated corresponds exactly to the real one. The vehicle has not committed any traffic violations.

\subsection{Second study case: speed calculation II}

This situation is similar to that of the previous example. In this case, the controlled vehicle traveled at a constant speed of $120 \mathrm{~km} / \mathrm{h}$. The images were captured the same day, half an hour later than the previous ones. The place and weather conditions were the same, thus the reference image is the same one used in the previous example. All configuration data shown in the previous example has remained unchanged. Once the parameters are correctly

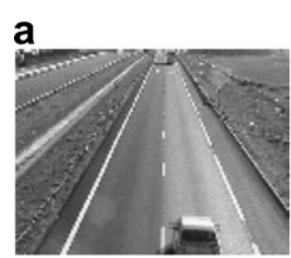

b
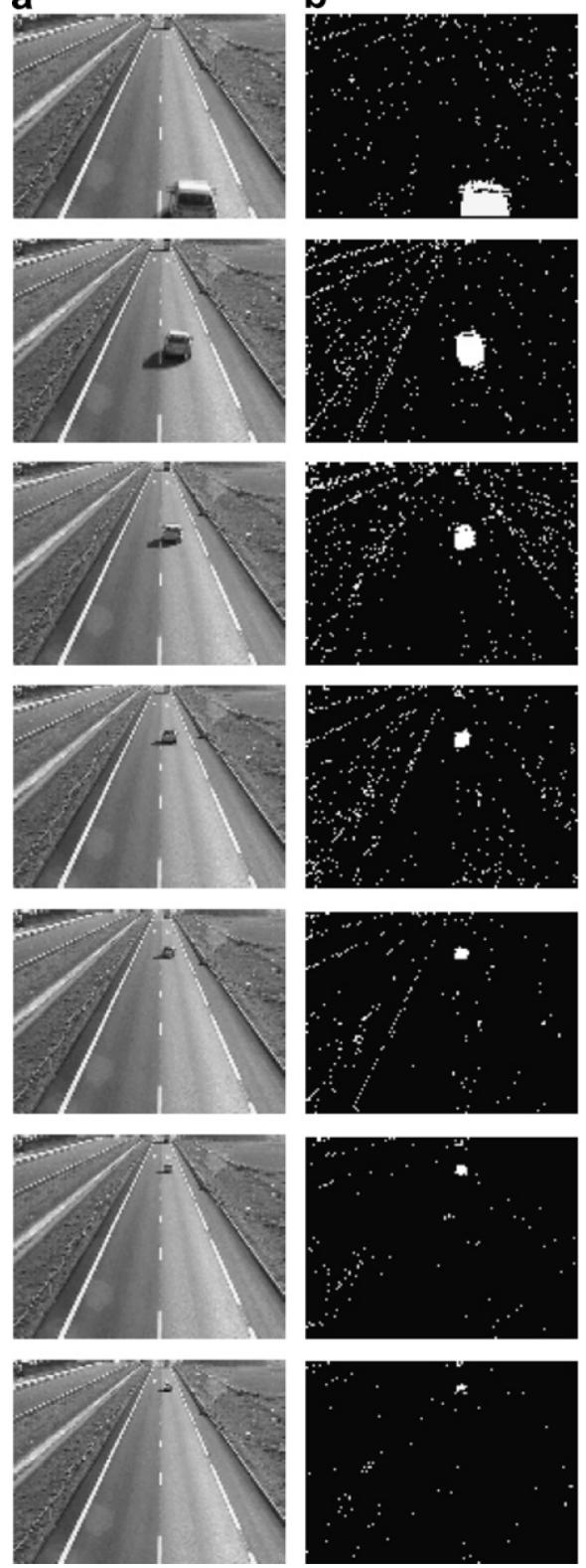

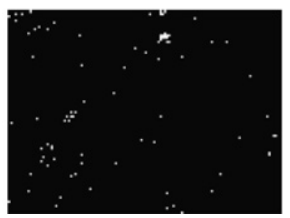

c
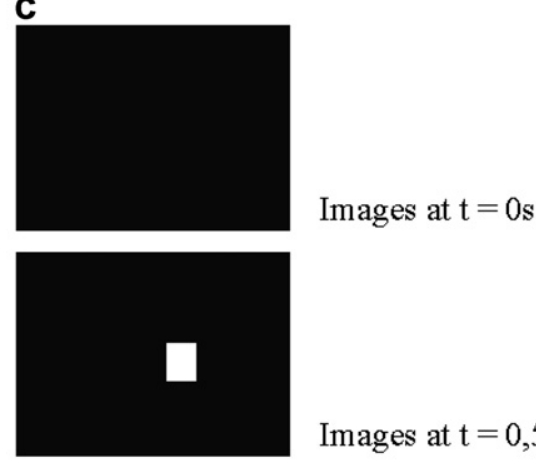

Images at $\mathrm{t}=0,5 \mathrm{~s}$

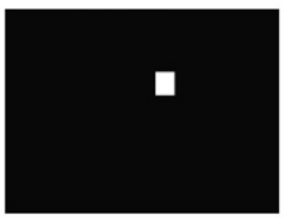

Images at $\mathrm{t}=1 \mathrm{~s}$

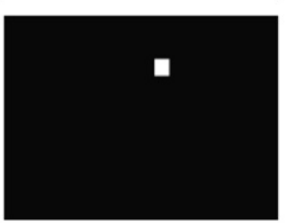

Images at $\mathrm{t}=1,5 \mathrm{~s}$

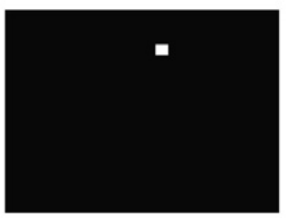

Images at $\mathrm{t}=2 \mathrm{~s}$

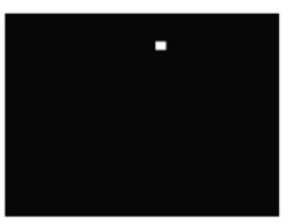

Images at $\mathrm{t}=2,5 \mathrm{~s}$

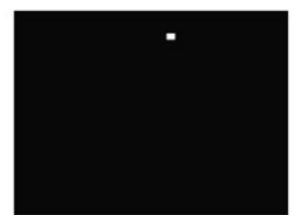

Images at $\mathrm{t}=3 \mathrm{~s}$

Fig. 9. Example 2: speed calculation II. 
established, they are checked to see if the application is capable of maintaining high efficiency in different situations. The results obtained in this case are those offered in Fig. 9. A car is detected in the right lane traveling at $122 \mathrm{~km} / \mathrm{h}$. These results practically agree with reality, since the real speed was $120 \mathrm{~km} / \mathrm{h}$. The deviation in the speed calculation is around $1.6 \%$. As for incidents, the car is detected to be speeding. The incident is only recorded in the image where it is detected.

\subsection{Third study case: mixed traffic}

In this case, a typical traffic scene with several types of vehicles is analyzed. The weather conditions were partly cloudy with strong winds. Furthermore, the date in which the images were processed is specified as a restricted-traffic date in order to see how the incident detection tool works.

Next, the sequences of images (real, segmented and processed) in this study case are shown as examples 3, 4 and 5 . The traffic parameters obtained in this set of examples 3, 4 and 5 - shown in Figs. 10-12, respectively - are: four vehicles traveling in the right lane at an average speed of $121 \mathrm{~km} / \mathrm{h}$ and only one traveling in the left lane at $129 \mathrm{~km} / \mathrm{h}$. According to the type of vehicle: three vehicles traveled at an average speed of $141 \mathrm{~km} / \mathrm{h}$ and two heavy vehicles at an average speed of $96 \mathrm{~km} / \mathrm{h}$. Five vehicles traveled altogether at an average speed of $123 \mathrm{~km} / \mathrm{h}$. Two heavy vehicles are detected on a restricted date. Moreover,
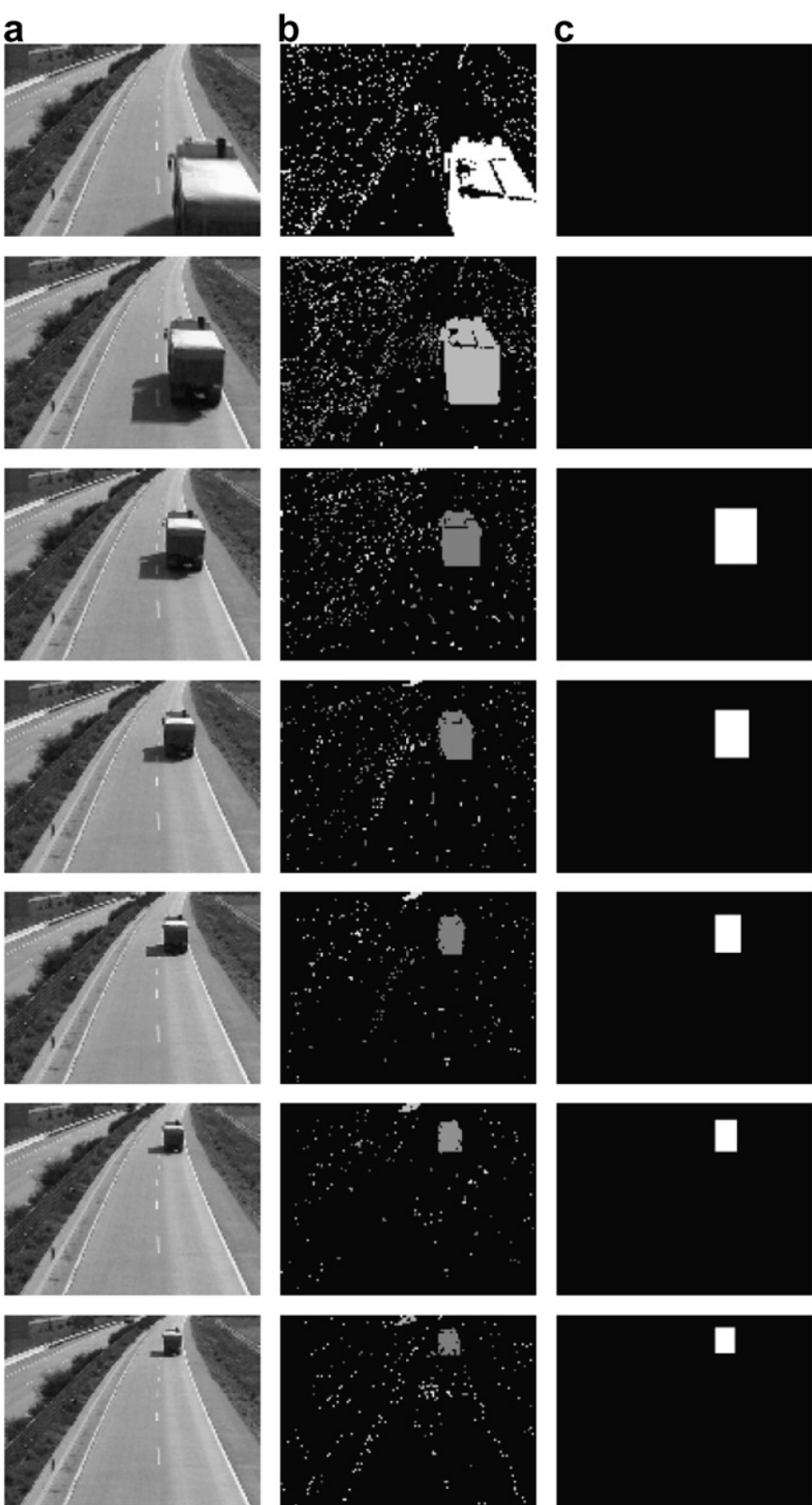
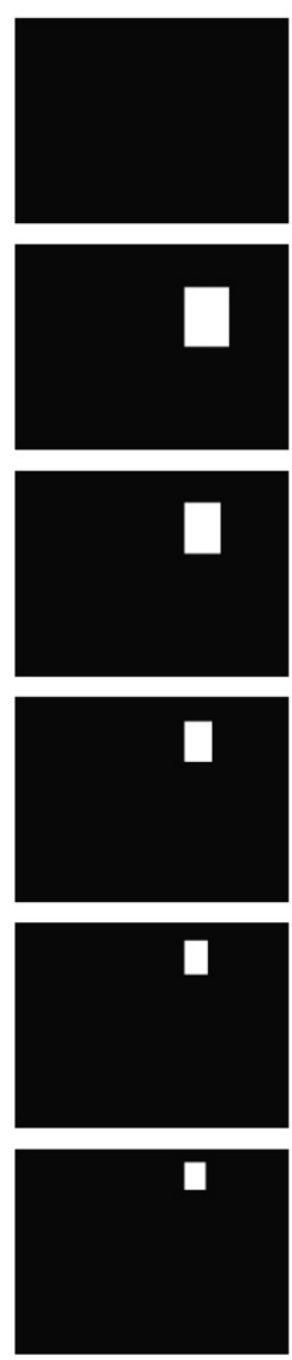

Images at $\mathrm{t}=3 \mathrm{~s}$

Images at $t=1 \mathrm{~s}$

Images at $\mathrm{t}=2 \mathrm{~s}$
Images at $\mathrm{t}=0 \mathrm{~s}$

Images at $\mathrm{t}=0,5 \mathrm{~s}$

Images at $\mathrm{t}=1,5 \mathrm{~s}$

Images at $\mathrm{t}=2,5 \mathrm{~s}$

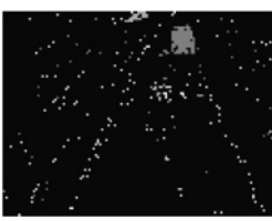

Fig. 10. Example 3. 
the following vehicles were speeding: the heavy vehicle matched with the yellow one in the processed image, which was traveling in the right lane and the cars matched with the red, gray and blue ones in the processed images.

\subsection{Fourth study case: vehicle overlapping}

In this section, a special vehicular transit case is portrayed, in which a vehicle is overlapped by another one in the image. In other words, there is no separation between them, from the camera's perspective. The images were taken in the same place, but a few minutes after those in the fourth study case, thus the configuration is the same. Notice that, in the sequence of images (see Fig. 13), both vehicles merge and take the color of the overlapping vehicle. From this point on, it is considered a single moving object. The application reports this situation by activating the message "Overlapping/Concealment". a
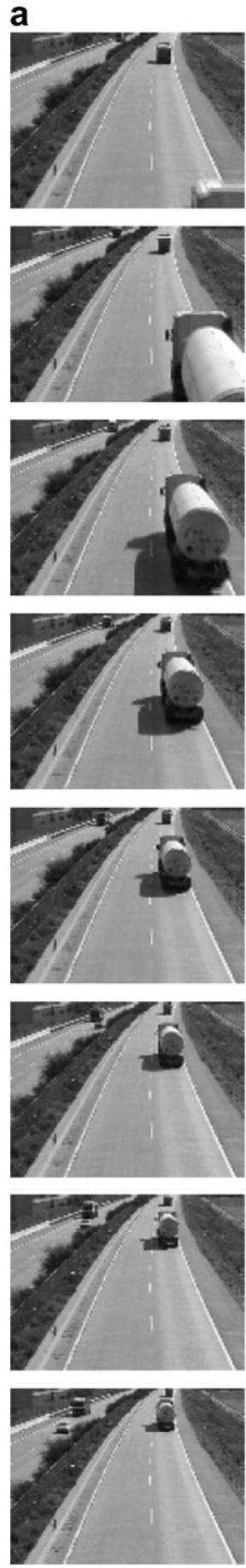

b
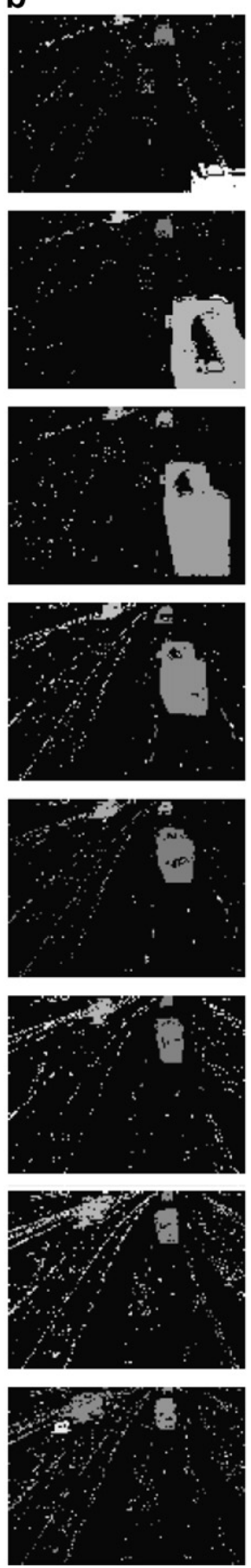

C

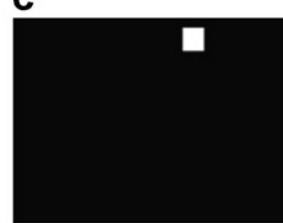

Images at $\mathrm{t}=3,5 \mathrm{~s}$

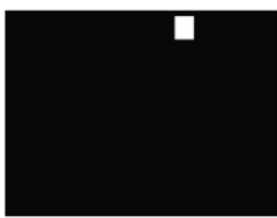

Images at $\mathrm{t}=4 \mathrm{~s}$

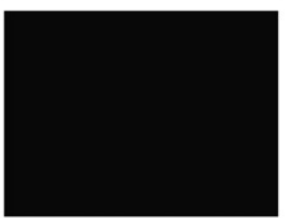

Images at $\mathrm{t}=4,5 \mathrm{~s}$

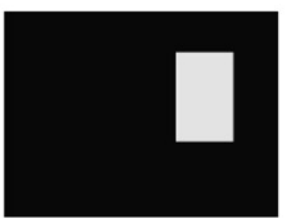

Images at $\mathrm{t}=5 \mathrm{~s}$

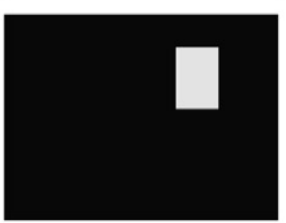

Images at $\mathrm{t}=5,5 \mathrm{~s}$

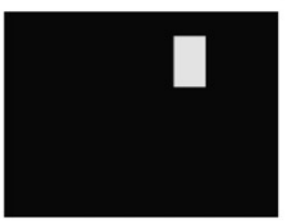

Images at $\mathrm{t}=6 \mathrm{~s}$

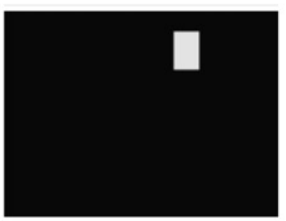

Images at $\mathrm{t}=6,5 \mathrm{~s}$

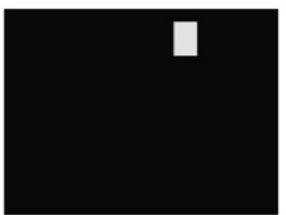

Images at $\mathrm{t}=7 \mathrm{~s}$

Fig. 11. Example 4. 


\subsection{Fifth study case: vehicle concealment}

In this case, a vehicle comes between the camera and another vehicle, hindering its visibility. To see how the application performs in this situation, a sequence of images corresponding to the same place and practically the same time as those in the fourth study case, was taken (see Fig. 14). As we can see in the sequence, the heavy yellow vehicle is concealing the white car. This concealment becomes real in the image corresponding to moment of time $t=2 \mathrm{~s}$. From here on, only the heavy vehicle is considered, since the car cannot be seen. The application informs of this the same way as in the previous example, which is by activating the message "Overlapping/Concealment".

\subsection{Sixth study case: solid line overstepping and strange movement}

To represent these two incidents, an image in the sequence of images corresponding to the first example is
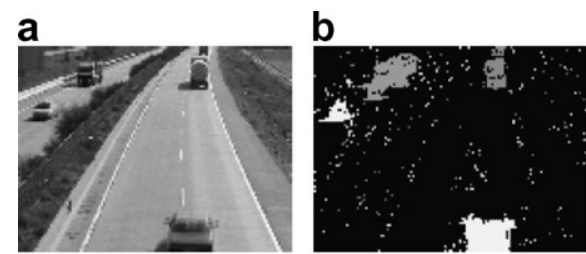

C
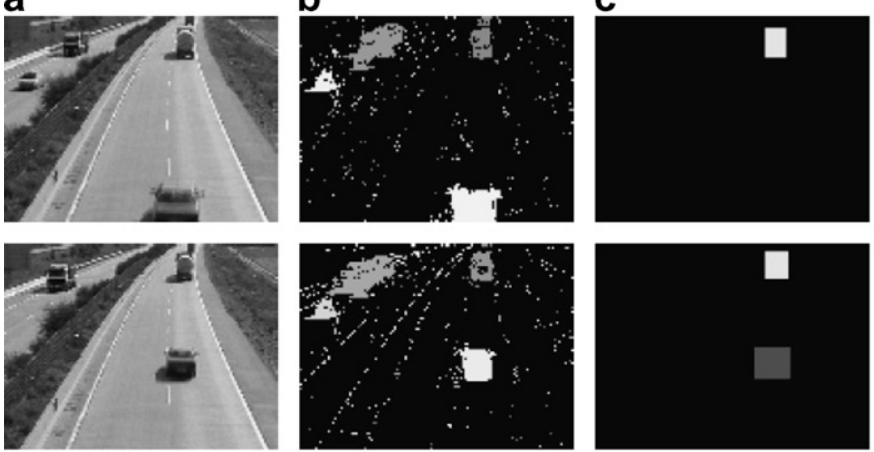

Images at $\mathrm{t}=7,5 \mathrm{~s}$
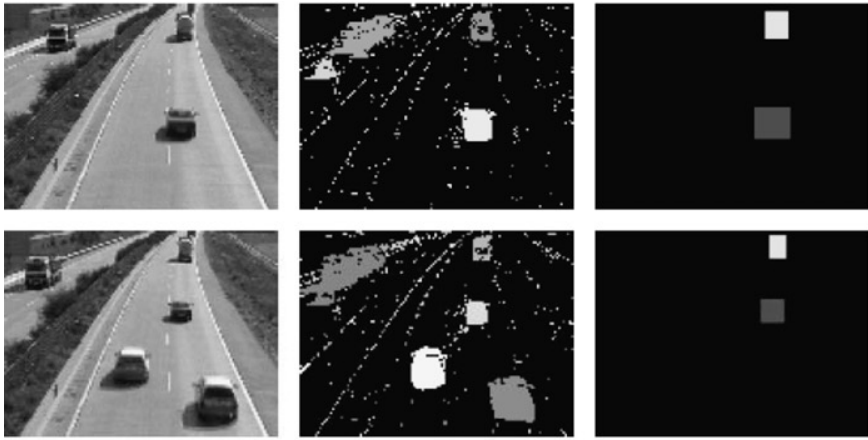

Images at $\mathrm{t}=8 \mathrm{~s}$
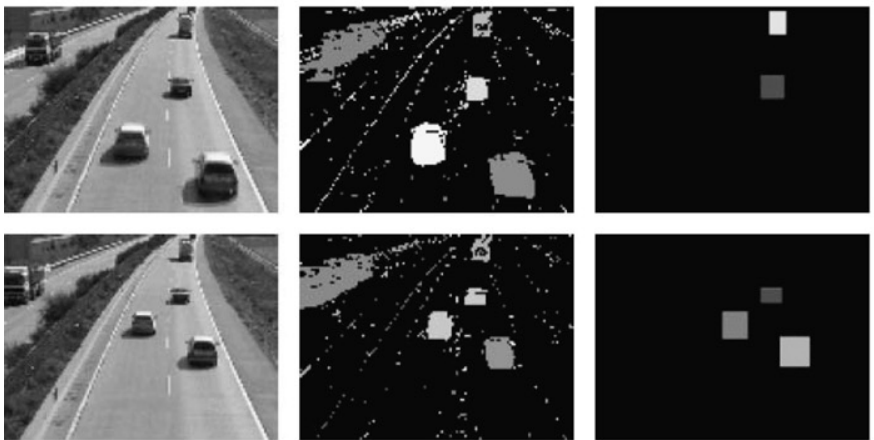

Images at $\mathrm{t}=8,5 \mathrm{~s}$
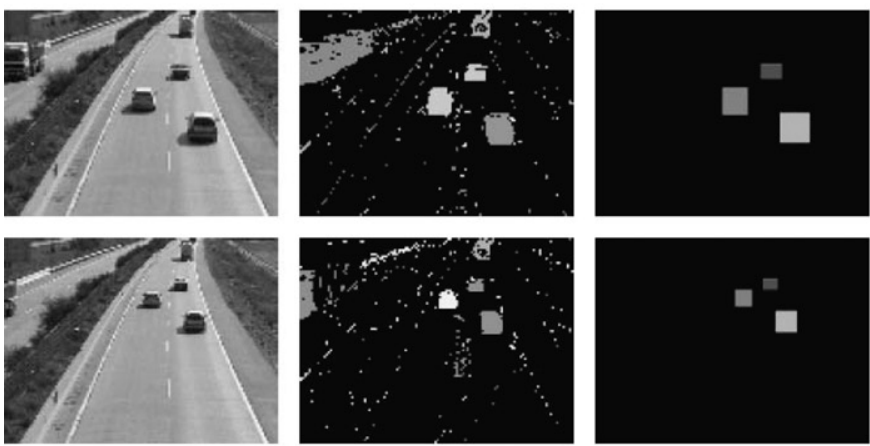

Images at $t=9 \mathrm{~s}$
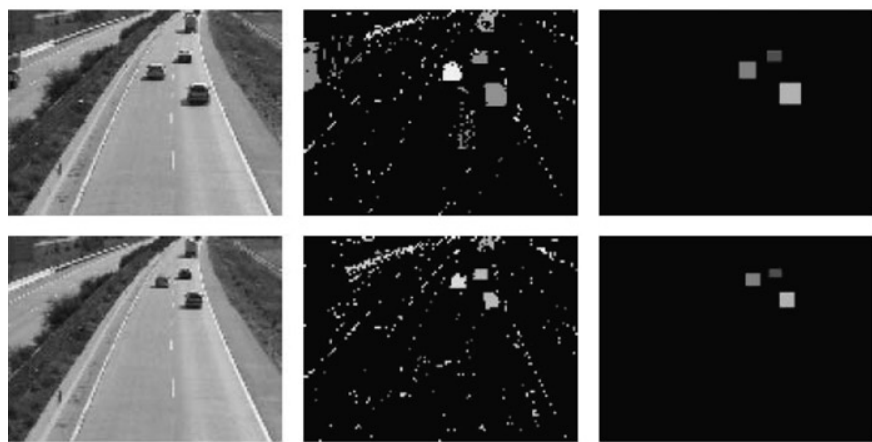

Images at $\mathrm{t}=9,5 \mathrm{~s}$
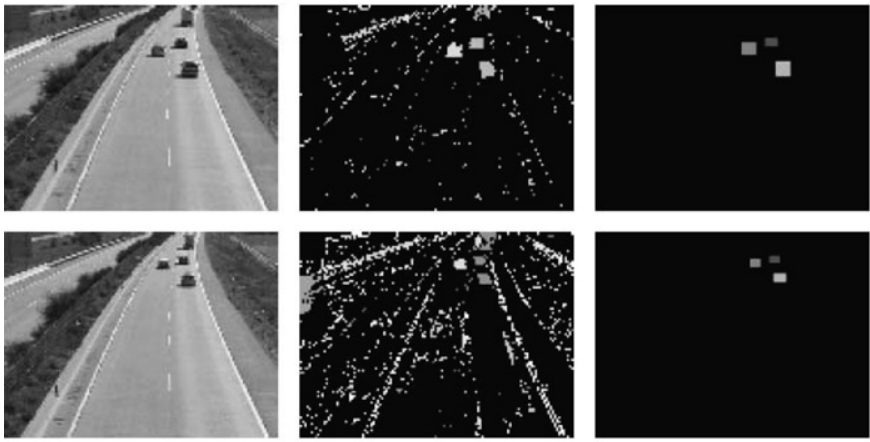

Images at $\mathrm{t}=10 \mathrm{~s}$
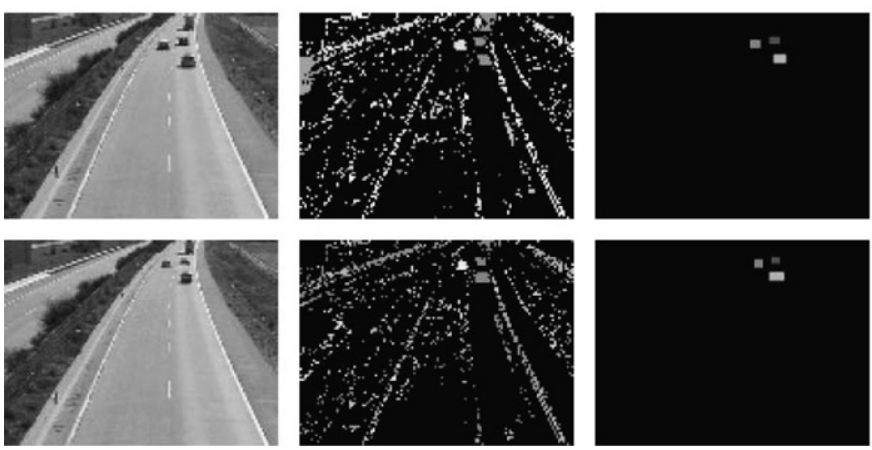

Images at $\mathrm{t}=10,5 \mathrm{~s}$

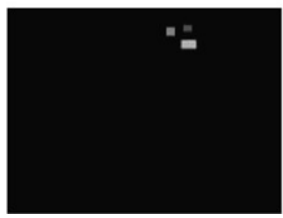

Images at $\mathrm{t}=11 \mathrm{~s}$

Fig. 12. Example 5. 
a
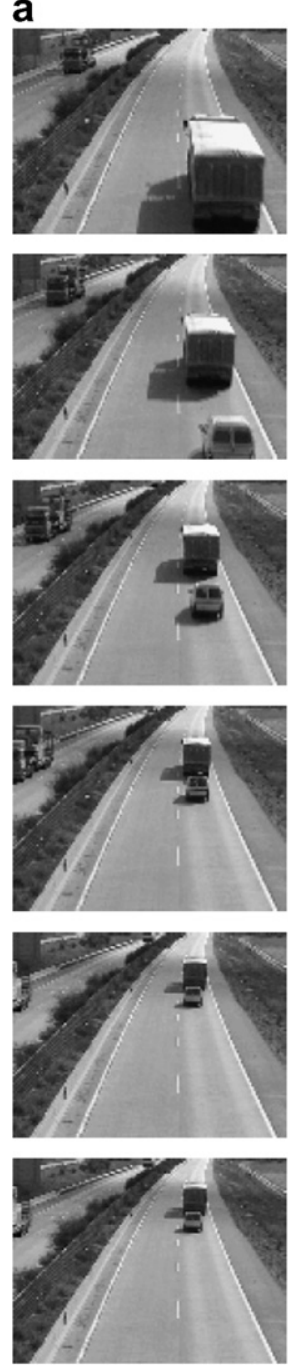

b
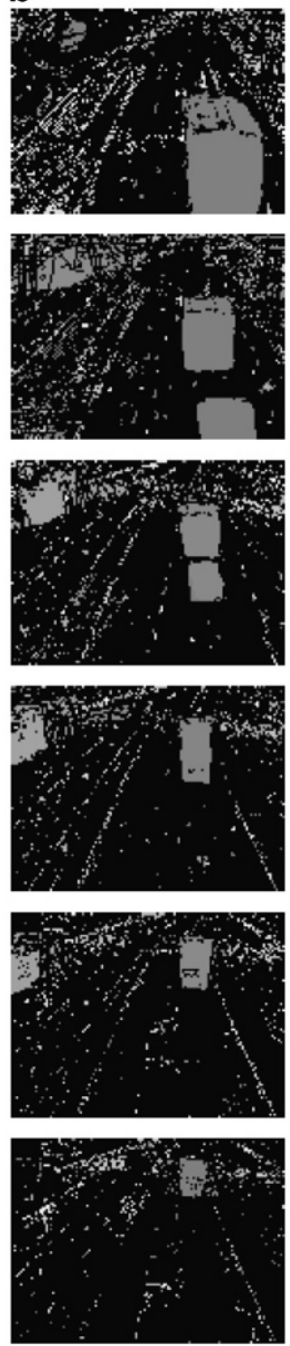

C

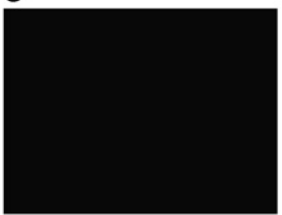

Images at $\mathrm{t}=0 \mathrm{~s}$

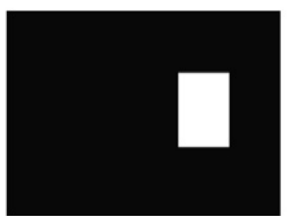

Images at $\mathrm{t}=0,5 \mathrm{~s}$

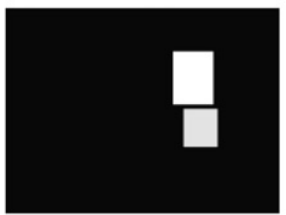

Images at $t=1 \mathrm{~s}$

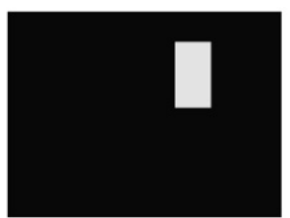

Images at $\mathrm{t}=1,5 \mathrm{~s}$

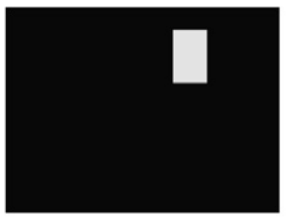

Images at $\mathrm{t}=2 \mathrm{~s}$

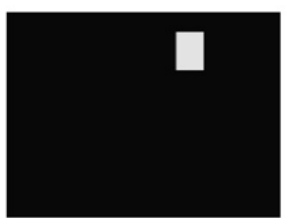

Images at $\mathrm{t}=2,5 \mathrm{~s}$

Fig. 13. Example 6: vehicle overlapping.

modified. To be more specific the fourth image, the one corresponding to moment of time $t=1.5 \mathrm{~s}$, has been changed in regard to its order. The vehicle's location has been modified by changing lanes and its vertical position has been reduced to simulate a movement of the vehicle which is too abrupt. The configuration is the same here as in the first example, except for a three-pixel-thick line representing a solid line, which has been drawn in the reference image. In this example, the speed control has been removed because, as a result of modifying an image in the sequence, the speed of the vehicle may not match the real one. Furthermore, this example only focuses on showing how well the application works regarding speeding and strange movement incidents. We will now show the sequences of images for this study case in Fig. 15. The incidents detected in this case are the following: in the fourth image of the sequence, the vehicle has overstepped the solid line and it has traveled across too far (when going from the right to the left lane). In the fifth image, the vehicle has overstepped the solid line again, when going from the right to the left lane.

\section{Conclusions}

This paper has introduced a knowledge-driven system capable of controlling traffic on highways and freeways in one direction of traffic. At maximum, the application controls three lanes of regular traffic (right, middle and left) and an entrance/exit ramp. The images are captured by a video camera mounted on top of a bridge, digitized and transferred to a moving objects recognition system. Through segmentation, this system provides a sequence of images where the movements of the objects in the scene are shown. The application developed eliminates noise in the segmented images and it detects moving objects through a sequential run of the pixels in those images. The position of each object on the road is calculated and the objects are classified according to the categories of valid type of vehicle (motorcycle, car, passenger and cargo vehicle and heavy vehicle) or to strange object category. Up to this point in the processing, the application can detect the following traffic incidents: presence of a strange object on 
a
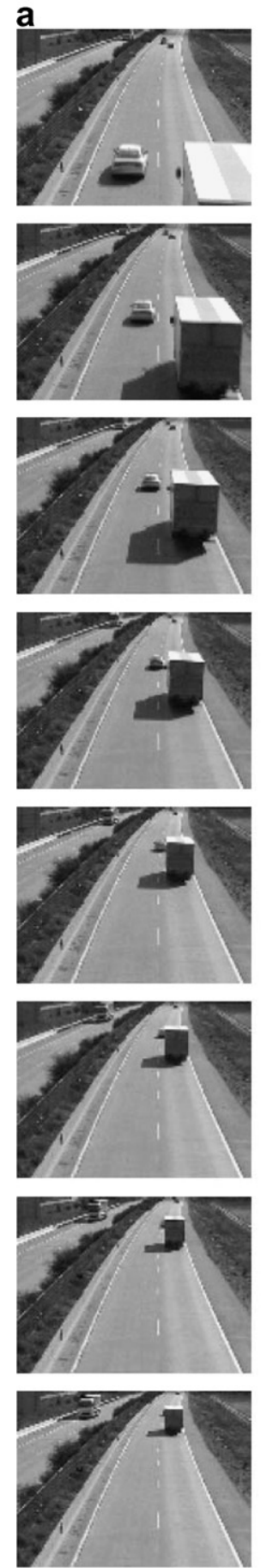
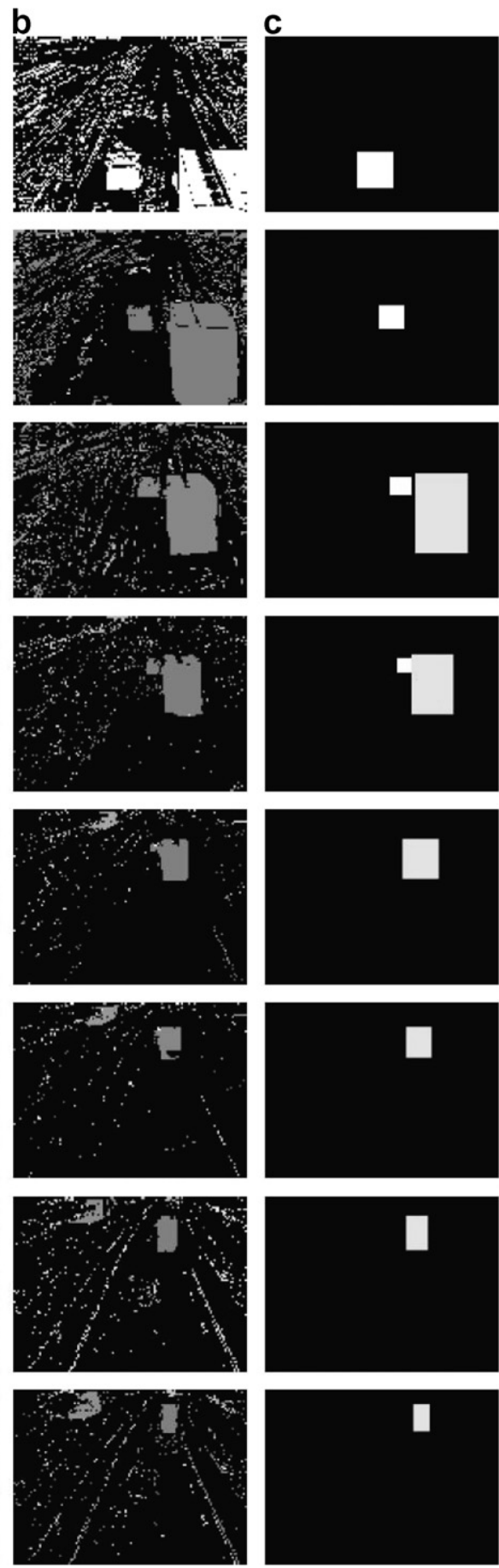

Fig. 14. Example 7: vehicle concealment.
Images at $\mathrm{t}=0 \mathrm{~s}$

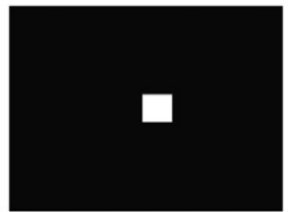

Images at $\mathrm{t}=0,5 \mathrm{~s}$

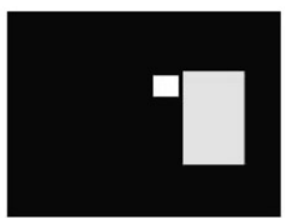

Images at $\mathrm{t}=1 \mathrm{~s}$

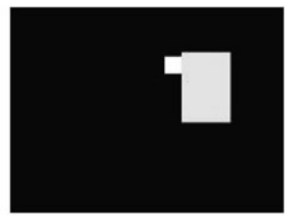

Images at $\mathrm{t}=1,5 \mathrm{~s}$

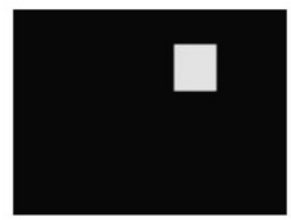

Images at $\mathrm{t}=2 \mathrm{~s}$

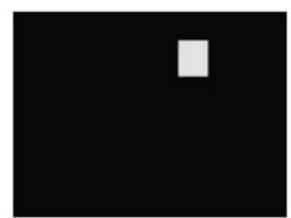

Images at $\mathrm{t}=2,5 \mathrm{~s}$

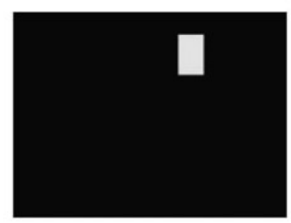

Images at $\mathrm{t}=3 \mathrm{~s}$

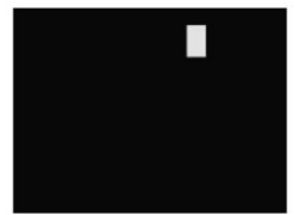

Images at $\mathrm{t}=3,5 \mathrm{~s}$ the road, heavy vehicle traveling on restricted dates and vehicle traveling on the shoulder.

By analyzing the current and previous segmented images, we can determine the movement of each vehicle in the scene. The application has the capability of matching these vehicles in such a way that vehicles in the previous segmented image are matched to those in the current one. Each match defines a single real vehicle, represented by the position it occupied in the previous image and the one it occupies in the current image. The speed of the current vehicles that have been matched is calculated. The number of vehicles that have entered the scene is determined and they are classified according to type of vehicle and position. For each vehicle that has disappeared from the scene, we determine whether it has really left the scene, it has been overlapped or it has been concealed by another vehicle. Likewise, by analyzing motion, the application can detect the following incidents: a vehicle speeding or driving 

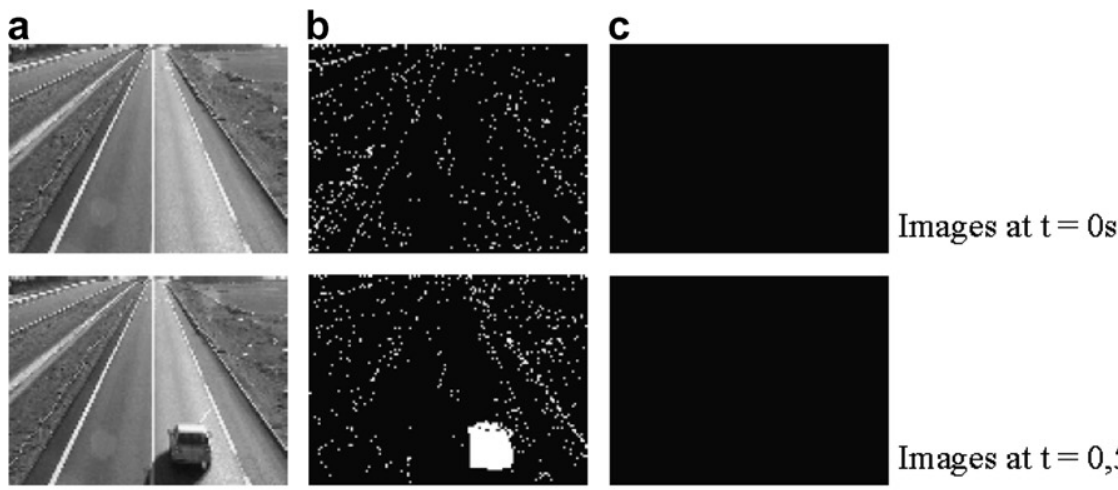

Images at $\mathrm{t}=0,5 \mathrm{~s}$
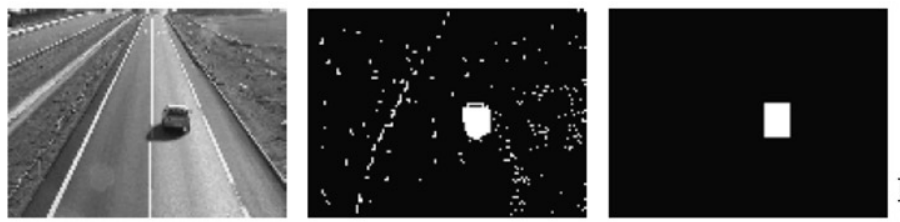

Images at $\mathrm{t}=1 \mathrm{~s}$
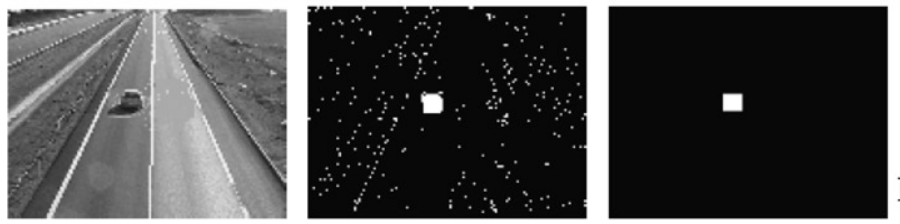

Images at $\mathrm{t}=1,5 \mathrm{~s}$
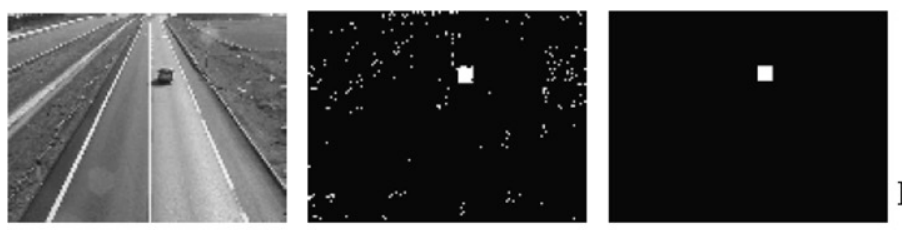

Images at $\mathrm{t}=2 \mathrm{~s}$
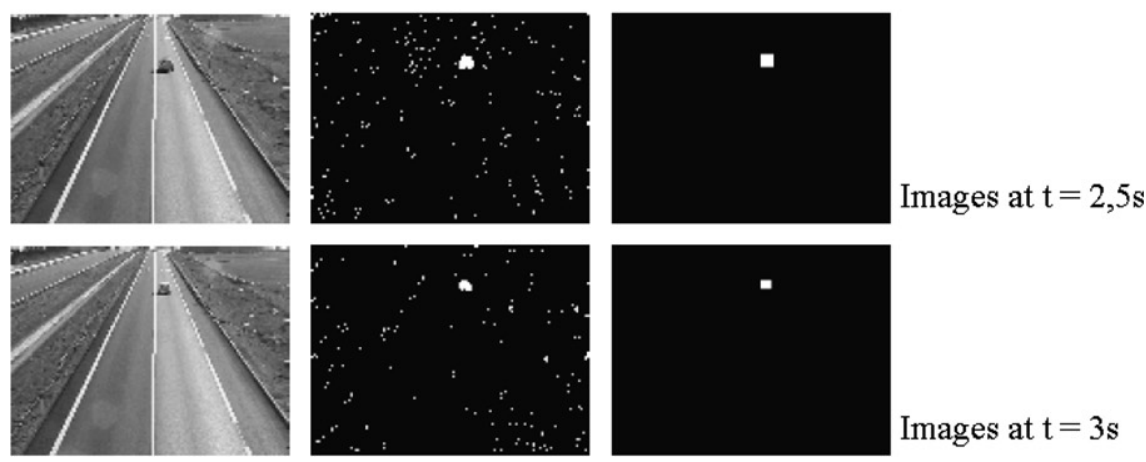

Images at $\mathrm{t}=3 \mathrm{~s}$

Fig. 15. Example 8: solid line overstepping and strange movement.

too slowly, a stopped vehicle, congestion on the road, a vehicle overstepping the solid line and a vehicle's strange movement. The application is ready to determine the number of vehicles that have appeared in the scene during the time of the analysis of the sequence of images, classifying this information according to type of vehicle and the lane where it is traveling. It can also offer a summary of all incidents detected.

We can summarize the functionality of the implemented system's performance by saying that vehicle identification, classification and positioning on the road mostly agree with reality. The small disagreements in measuring speed are more than acceptable and could probably be corrected through the introduction of more distance zones. For this reason, the parameters offered by the system are very much in harmony with what happens on real roads. Furthermore, controlling incidents, which were expected to be identified from the beginning, is done in a fast and ideal way, without lowering the system's performance or proper running. On the other hand, everything has been achieved with the idea and purpose of doing away with difficult mathematical calculations or functions. Thus, quick conceptualization of the process is possible and the burden of computation is reduced.

Another great advantage is the use of very few configuration parameters, which makes configuration quick and easy. Also, the fact that once these parameters are accurately defined, they can be used for all traffic in the scene 
and do not have to be altered for the system to work properly. In short, we have been able to implement a quick, easy-to-use-and-configure system, which offers a wide range of traffic flow data and complies with the system's initial specifications.

\section{Acknowledgements}

This work is supported in part by the Spanish CICYT TIN2004-07661-C02-02 and TIN2007-67586-C0202 Grants, and the Junta de Comunidades de Castilla-La Mancha PBI06-0099 Grant.

\section{References}

Abramczuk, T. (1984). A microcomputer based TV detector for road traffic. In Symposium on road research program, Tokyo, Japan.

Ali, A. T., \& Dagless, E. L. (1991). Computer vision-aided road traffic monitoring. In Proceedings ISATA 24th international conference on road transport informatics and intelligent vehicle-highway systems.

Ali, A. T., \& Dagless, E. L. (1992). A parallel processing model for realtime computer vision-aided road traffic monitoring. Parallel Processing Letters, 2(2), 257-264.

Blosseville, J. M. (1990). Image processing for traffic management. France: Traffic and Control Research Unit, INRETS.

Blosseville, J. M., Krafft, C., Lenoir, F., Motyka, V., \& Beucher, S. (1989). TITAN: A traffic measurement system using image processing techniques. In IEEE 2nd international conference on road monitoring (pp. 84-88).

Briquet, P. (1992). Video processing applied to road and urban traffic monitoring. In 6th international conference on road traffic monitoring (pp. 153-157).

Badenas, J., Bober, M., \& Pla, F. (2001). Segmenting traffic scenes from grey level and motion information. Pattern Analysis \& Applications, 4, 28-38.

Badenas, J., Sanchiz, J. M., \& Pla, F. (1999). Motion-based segmentation and region tracking in image sequences. Pattern Recognition, 34(3), 661-670.

Barni, M., Bartolini, F., Capellini, V., Lambardi, F., \& Piva, A. (1998). Fuzzy motion detection for highway traffic control. In Workshop on advanced video-based surveillance systems (pp. 58-66).

Beymer, B., McLauchlan, P., Coifman, B., \& Malik, J. (1997). A real-time computer vision system for measuring traffic parameters. Computer Vision and Pattern Recognition, 495-501.

Bulas-Cruz, J., Ali, A. T., \& Dagless, E. L. (1993). A temporal smoothing technique for real-time motion detection. Computer Analysis of Images and Patterns, 379-386.

Cornish, M. T., \& Wakefield, J. P. (1996). Automatically locating an area of interest and maintaining a reference image to aid the real-time tracking of objects. In Proceedings of the British machine vision conference (pp. 475-484).

Cucchiara, R., Piccardi, M., \& Mello, P. (2000). Image analysis and rulebased reasoning for a traffic monitoringsystem. IEEE Transactions on Intelligent Transportation Systems, 1(2), 119-130.

Dickinson, K. W., \& Wan, C. L. (1989). Road traffic monitoring using the TRIP II system. In: 2nd International conference on road traffic monitoring (pp. 56-60).

Dubuisson, M., \& Jain, A. (1995). Contour extraction of moving objects in complex outdoor scenes. International Journal of Computer Vision, $14,83-105$.

Fathy, M., \& Siyal, M. Y. (1995). A real-time image processing approach to measure traffic queue parameters. IEE Proceedings Vision, Image and Signal Processing, 142(5), 297-303.

Fernández, M. A., Fernández-Caballero, A., López, M. T., \& Mira, J. (2003). Length-Speed Ratio (LSR) as a characteristic for moving elements real-time classification. Real-Time Imaging, 9(1), 49-59.
Fernández-Caballero, A., Mira, J., Fernández, M. A., \& López, M. T. (2001). Segmentation from motion of non-rigid objects by neuronal lateral interaction. Pattern Recognition Letters, 22(14), 1517-1524.

Fernández-Caballero, A., Fernández, M. A., Mira, J., \& Delgado, A. E. (2003). Spatio-temporal shape building from image sequences using lateral interaction in accumulative computation. Pattern Recognition, 36(5), 1131-1142.

Fernández-Caballero, A., Mira, J., Delgado, A. E., \& Fernández, M. A. (2003). Lateral interaction in accumulative computation: A model for motion detection. Neurocomputing, 50, 341-364.

Gil, S., \& Punt, T. (1993). Non-linear multiresolution relaxation for alerting. In Proceedings of the European conference on circuit theory and design (pp. 1639-1644).

Gupte, S., Masoud, O., Martin, R. F. K., \& Papanikiolopoulos, N. P. (2002). Detection, and classification of vehicles. IEEE Transactions on Intelligent Transportation Systems, 3(1), 37-47.

Ha, D. M., Lee, J. M., \& Kim, Y. D. (2004). Neural-edge-based vehicle detection and traffic parameter extraction. Image and Vision Computing, 22(11), 899-907.

Hoose, N. (1989). Queue detection using computer image processing. In 2nd international conference on road traffic monitoring (pp. 94 98).

Inigo, R. M. (1989). Application of machine vision to traffic monitoring and control. IEEE Transactions on Vehicular Technology, 38(3), $112-122$.

Inigo, R. M. (1985). Traffic monitoring and control using machine vision: A survey. IEEE Transactions on Industrial Electronics, 32(3), 177185.

Ji, X., Wei, Z., \& Feng, Y. (2006). Effective vehicle detection technique for traffic surveillance systems. Journal of Visual Communication and Image Representation, 17(3), 647-658.

Kastrinaki, V., Zervakis, M., \& Kalaitzakis, K. (2003). A survey of video processing techniques for traffic applications. Image and Vision Computing, 21(4), 359-381.

Kelly, D. M. (1992). Results of field trial of the IMPACTS image processing system for traffic monitoring. In 6th international conference on road traffic monitoring (pp. 137-142).

Koller, D., Weber, J., \& Malik, J. (1994). Robust multiple car tracking with occlusion reasoning. In Proceedings 5th European conference on computer vision (pp. 189-196).

Koller, D., Weber, J., Huang, T., Malik, J., Ogasawara, G., Rao, B., et al. (1994). Towards robust automatic traffic scene analysis in real-time. In International conference on pattern recognition (pp. 126-131).

López, M. T., Fernández-Caballero, A., Fernández, M. A., Mira, J., \& Delgado, A. E. (in press). Dynamic visual attention model in image sequences. Image and Vision Computing.

López, M. T., Fernández-Caballero, A., Fernández, M. A., Mira, J., \& Delgado, A. E. (2006). Visual surveillance by dynamic visual attention method. Pattern Recognition, 39(11), 2194-2211.

López, M. T., Fernández-Caballero, A., Mira, J., Delgado, A. E., \& Fernández, M. A. (2006). Algorithmic lateral inhibition method in dynamic and selective visual attention task: Application to moving objects detection and labelling. Expert Systems with Applications, 31(3), 570-594.

Malik, J., Weber, J., Luong, Q. T., \& Koller, D. (1995). Smart cars and smart roads. In Proceedings of the 6th British machine vision conference (pp. 367-381).

Mira, J., Delgado, A. E., Fernández-Caballero, A., \& Fernández, M. A. (2004). Knowledge modelling for the motion detection task: The algorithmic lateral inhibition method. Expert Systems with Applications, 27(2), 169-185.

Proper, A. T. (1999). Intelligent transportation system benefits: 1999 update. US Department of Transportation, Washington, DC.

Sonka, M., Hlavac, V., \& Boyle, R. (1993). Image processing, analysis and machine vision. Chapman \& Hall.

Takaba, S., Sakauchi, M., Kaneko, T., Hwang, B. W., \& Sekine, T. (1982). Measurement of traffic flow using real-time processing of moving pictures. In 32nd conference on vehicular technology (pp. 488-494). 
Teal, M., \& Ellis, T. (1996). Spatial-temporal reasoning on object motion. In Proceedings of the British machine vision conference (pp. 465-474). BMVA Press.

Versavel, J., Lemaire, F., \& van der Stede, D. (1989). Camera and computer-aided traffic sensor. In 2nd international conference on road traffic monitoring (pp. 66-70).
Waterfall, R., \& Dickinson, K. (1984). Image processing applied to traffic. Traffic Engineering Control, 25, 60-67.

Zhu, Z., Xu, G., Yang, B., Shi, D., \& Lin, X. (2000). VISATRAM: A realtime vision system for automatic traffic monitoring. Image and Vision Computing, 18(10), 781-794. 